\title{
Normal-conducting scaling fixed field alternating gradient accelerator for proton therapy
}

\author{
J. M. Garland, R. B. Appleby, ${ }^{\dagger}$ H. Owen, and S. Tygier \\ University of Manchester, Manchester, M13 9PL, United Kingdom and Cockcroft Institute, \\ Warrington, WA4 4AD, United Kingdom
}

(Received 28 April 2015; published 29 September 2015)

\begin{abstract}
In this paper we present a new lattice design for a 30-350 MeV scaling fixed-field alternating gradient accelerator for proton therapy and tomography-NORMA (NOrmal-conducting Racetrack Medical Accelerator). The energy range allows the realization of proton computed tomography and utilizes normal conducting magnets in both a conventional circular ring option and a novel racetrack configuration, both designed using advanced optimization algorithms we have developed in Pyzgoubi. Both configurations consist of ten focusing-defocusing-focusing triplet cells and operate in the second stability region of Hills equation. The ring configuration has a circumference of $60 \mathrm{~m}$, a peak magnetic field seen by the beam of $<1.6 \mathrm{~T}$, a maximum horizontal orbit excursion of $44 \mathrm{~cm}$ and a dynamic aperture of $68 \mathrm{~mm} \mathrm{mrad-}$ determined using a novel dynamic aperture (DA) calculation technique. The racetrack alternative is realized by adding magnet-free drift space in between cells at two opposing points in the ring, to facilitate injection and extraction. Our racetrack design has a total magnet-free straight lengths of $4.9 \mathrm{~m}$, a circumference of $71 \mathrm{~m}$, a peak magnetic field seen by the beam of $<1.74 \mathrm{~T}$, a maximum horizontal orbit excursion of $50 \mathrm{~cm}$ and a DA of $58 \mathrm{~mm}$ mrad. A transverse magnet misalignment model is also presented for the ring and racetrack configurations where the DA remains above $40 \mathrm{~mm}$ mrad for randomly misaligned error distributions with a standard deviation up to $100 \mu \mathrm{m}$.
\end{abstract}

DOI: 10.1103/PhysRevSTAB.18.094701

PACS numbers: 29.20.D-, 29.27.-a, 87.56.bd, 87.57.Q-

\section{INTRODUCTION}

External beam radiotherapy, which is predominantly used for the treatment of cancer, is mostly carried out using x-rays generated in electron linacs. In the UK there are around 130,000 radiotherapy treatments each year [1]. Modern x-ray delivery techniques such as intensitymodulated radiotherapy (IMRT) give an excellent conformality of the delivered dose to a desired treatment volume, which is aided by directing the delivered $\mathrm{x}$-rays into the patient from a variety of different angles.

However, it has been recognized since the 1940s that heavier particles such as protons may deliver a more localized deposition of dose due to their Bethe-Bloch slowing in matter [2]. The dose deposition rate is roughly inverse with the particle velocity, and this concentrates the total dose from an incident beam into a characteristic Bragg peak at a depth determined by the initial particle energy; for example a proton with an initial energy of $250 \mathrm{MeV}$ has a peak in dose deposition at a depth of around $38 \mathrm{~cm}$ in water, enough for adult treatments. The effect of straggling means

\footnotetext{
*james.garland@hep.manchester.ac.uk

robert.appleby@manchester.ac.uk
}

Published by the American Physical Society under the terms of the Creative Commons Attribution 3.0 License. Further distribution of this work must maintain attribution to the author(s) and the published article's title, journal citation, and DOI. that the depth spread of an incident monochromatic beam is approximately 1 percent of the total range [3]. The concentration of most of the delivered dose in the peak can spare a dose to surrounding healthy tissue if the intervening patient density is well enough known to be able to set the incident particle energy correctly. The present burgeoning method of proton delivery utilizes a narrow spot beam of particles that is scanned, both transversely and by varying the energy to vary the range, in a technique known as intensity-modulated proton therapy (IMPT) [3,4].

Adequate treatment of adult patients conventionally leads to a clinical requirement [5] on the incident proton energy of 70 to $250 \mathrm{MeV}$ (although some treatments nearer to the patient surface-such as treatment of the eye or skin-may require delivery of lower-energy protons), which are available via a range of particle accelerator technologies. Clinical centers today utilize either: cyclotrons, in which the effectively fixed extracted proton energy is adjusted for treatment by using a variable degrader arrangement; or synchrotrons, in which the extracted energy may be varied every magnet cycle albeit at a rate that is typically a few seconds long [4] (it should be noted that designs for rapidcycling medical synchrotrons exist that propose cycling times as short as $20 \mathrm{~ms}$ or even less) [6,7].

In either case, the clinical specification to deliver around 1 Gray to a 1 litre treatment volume in 1 minute translates to around $1 \times 10^{11}$ protons per fraction, or equivalently a little 
over $0.1 \mathrm{nA}$ of effective proton current incident at the patient. To make the best use of the Bragg peak it is desirable to vary the proton energy in steps of $5 \mathrm{MeV}$ or less and to have an energy spread somewhat smaller than this [5]. Similarly, it is desirable to have an incident spot size at the patient of a few millimetres or less, which results in a beam emittance requirement of less than $40 \mathrm{~mm}$ mrad. Throughout this document, emittance and acceptance (dynamic aperture) are given as $\epsilon$ where the area in phase space $A=\pi \epsilon$. Ideally one would also like to vary the energy pulse by pulse at up to $1 \mathrm{kHz}$ to make use of dose repainting that such a variation in speed offers [3], but this is not readily achievable either in cyclotrons or synchrotrons in operation today.

Over fifty proton and carbon ion treatment centers now operate around the world that offer a mixture of IMPT and the older passive scattering treatment methods [8]. While they all offer excellent treatment capabilities, they rely on the accurate determination of patient density to set an accurate delivered proton range; without this there is a significant issue of potential overdosing of surrounding tissues or underdosing the treatment volume itself, both of which are more serious with protons due to the strong dose gradient at the end of the particle range [3]. Proton treatments are limited by the consequent need to allocate a margin around the clinical treatment volume to take account of this range uncertainty, analogous to that used in IMRT [9]. Patient density may be derived from a combination of a number of imaging methods such as $\mathrm{x}$-ray computed tomography (CT) or magnetic resonance imaging. CT in particular is limited in its accuracy by the need to translate from its Hounsfield units to the needed proton stopping power through the use of a conventional stoichiometric conversion [10]; this conversion can introduce range uncertainties of a few $\mathrm{mm}$ which are highly desirable to reduce [11].

Proton computed tomography (pCT) can reduce range uncertainty [12-14] as instead of X-rays it utilizes protons of high enough incident energy to pass through the patient. Knowledge of that incident energy and measurement of the residual energy afterwards allows tomographic reconstruction of the patient density if sufficient particles and incident angles are utilized, which can reduce the range uncertainty for treatment [15]. Suitable residual range and tracking instruments are under active development [16-19] and it is expected that clinical adoption will be in the next few years. However, whilst tomography can be carried out with $250 \mathrm{MeV}$ protons either in children or for specific parts of the patient (notably the head and neck), proton tomography of the entirety of the adult body requires incident proton energies up to as much as $350 \mathrm{MeV}$ [20]. Unfortunately, there is currently no stopped-beam clinical center where protons up to $350 \mathrm{MeV}$ are available (the Gatchina $1 \mathrm{GeV}$ synchrocyclotron [21] is a special case). Complications arise for cyclotrons above around $250 \mathrm{MeV}$, and synchrotrons are otherwise limited by their naturally slower cycle rate and extraction interval. The patient dose required to obtain a good pCT image is of the order of a few hundred protons per voxel. This translates to several million incident protons or a dose of several mGy, around three orders of magnitude smaller than the dose of several Gy delivered in each treatment fraction $[20,22]$. An accelerator capable of delivering $350 \mathrm{MeV}$ protons with variable final energy and intensity (and not requiring a degrader) would be an excellent source both for therapy and for pCT.

A class of accelerator originally developed in the 1950s - but enjoying a reemergence in recent years-is the fixed-field alternating-gradient accelerator (FFAG) [23], which is capable of addressing the above needs [24]. FFAGs are in principle capable both of obtaining extracted proton energies up to and beyond $350 \mathrm{MeV}$ and of delivering pulse-by-pulse extraction with varying energy at rates up to $1 \mathrm{kHz}$. A FFAG typically has a large acceptance and can cope with variable bunch charge which would be necessary for the different intensities needed for $\mathrm{pCT}$ and treatment, provided a suitable injector was used. A single FFAG may therefore in principle deliver beams both for particle therapy and for $\mathrm{pCT}$, with associated advantages in machine reliability arising from the use of fixed fields and from the lower particle losses due to not needing a degrader. FFAGs come in two distinct types-scaling and nonscaling - with circular demonstration machines of both types having been realized in Japan and the UK in the last 15 years [25-28]. Two notable circular FFAG designs aimed at particle therapy were the RACCAM (Recherche en ACCélérateurs et Applications Médicales) spiral scaling FFAG proposal [29], and the nonscaling PAMELA (Particle Accelerator for MEdicaL Applications) FFAG [30,31]. PAMELA proposes a combined proton and carbon-ion facility in which two compact superconducting FFAGs deliver protons from 70 to $250 \mathrm{MeV}$ from the smaller ring, and carbon ions from 110 to $450 \mathrm{MeV} / \mathrm{u}$ from the larger ring. The proton FFAG lattice consists of 12 focusing-defocusing-focusing (FDF) triplet cells where each superconducting magnet [32] has a number of harmonic components up to decapole that adjust the nonlinear, nonscaling focusing to minimize the variation of betatron tune with energy. The high field index and working point in the second stable region of Hill's equation $[33,34]$ allow for a small horizontal orbit excursion from injection to extraction (less than $20 \mathrm{~cm}$ ). Table I shows some principal parameters for PAMELA.

The possibility also exists to modify a ring by inserting several straights to ease injection and extraction. A common layout is to insert two long straights opposite each other to create a so-called racetrack lattice. Racetracks provide the potential to realize a more flexible lattice by offering longer magnet-free straight sections or other insertions, for example to suppress the horizontal dispersion [35]. With the aid of FFAG-like straight sections [36,37], which obey the scaling 
TABLE I. PAMELA parameters.

\begin{tabular}{lc}
\hline \hline Parameter (unit) & Value \\
\hline Energy range [MeV] & $70-250$ \\
Circumference [m] & 39.3 \\
Maximum drift [m] & 1.7 \\
Maximum field (F/D) [T] & $3.48 /-2.62$ \\
Magnet length/width [m] & $2 / 0.9$ \\
Horizontal orbit excursion [m] & 0.176 \\
Ring tune (horizontal/vertical) & $8.76 / 3.48$ \\
Field index $k$ & 36.7 \\
\hline \hline
\end{tabular}

relation, it is possible to develop a racetrack of arbitrary size that may realize many applications. For example, the nuSTORM (neutrinos from STORed Muons) FFAG [38] ring uses FFAG arc sections and FFAG straight sections to form a full FFAG racetrack design which facilitates the decay of pions to muons in the long straight sections, resulting in a beam of neutrinos for studying neutrino physics.

In this work we propose a new scaling FFAG design aimed specifically at proton therapy and $\mathrm{pCT}$ which accelerates protons from an injection energy of $30 \mathrm{MeV}$ to a maximum extracted energy of $350 \mathrm{MeV}$-higher than any dedicated proton therapy machine to date. Due to the complexity and expense of superconducting magnets we propose to use normal-conducting magnets with a maximum field $<1.8 \mathrm{~T}$ [39] and use a shaped pole face to realize the scaling law. The maximum field was arrived at in collaboration with magnet engineers [40] and takes into consideration FFAG magnets with optimized shaped pole-faces and the potential saturation of the iron of such magnets. Whilst superconducting magnets can have some advantages in terms of stability, the cost of designing a lattice which requires superconducting magnets can be high compared to normal-conducting technology. A full magnet design will allow accurate comparison between the two different types of technology and is currently being studied by the authors.

The larger footprint caused by the lower fields is minimized using numerical techniques to yield a lattice with only a modest increase in circumference over existing FFAG designs that give lower energy output such as the PAMELA proton ring. The optimization has been carried out with algorithms developed in Pyzgoubi [41] to minimize the lattice circumference whilst obtaining a given working point and dynamic aperture (DA). Our methods are computationally intensive but allow us to acquire a higher level of detail than has been previously reached. For example when examining the variation of DA in the tune space, our methods allow us to make more precise optimization of our parameters. Using the same methodology we have adapted the circular ring design into a racetrack configuration to provide additional space for injection, extraction and acceleration systems whilst not significantly impacting the overall circumference. In this paper we only consider magnet-free insertions in our ring design, and explore the limits of such a machine; in this paper we refer to such a machine as a racetrack.

The layout of this paper is as follows. In Sec. II we present the design process used to define the NORMA ring in terms of geometry, lattice parameters and performance and give details of the optimization procedures developed in Pyzgoubi. In Sec. II C the optimized layout and parameters of the NORMA ring option are presented. The NORMA ring layout and parameters are used as a starting point for Sec. III, where we present the racetrack NORMA design and optimization, and show the limits of the achievable machines. In Sec. IV we discuss possible rf acceleration, injection and extraction systems and finally in Sec. V we draw our conclusions.

\section{NORMA RING LATTICE}

In this section we describe the design procedure for the NORMA ring and justify our preliminary requirements. We first explain some geometric constraints and then describe our use of the simulation code Pyzgoubi [41], which utilizes the embedded tracking code zgoubi [42], to optimize the optics and dynamic aperture of the lattice. We conclude the section by giving the parameters and properties of the optimized NORMA ring lattice.

\section{A. Cell geometry and ring size}

An accelerator design intended for use in a hospital environment must be cost-effective in terms of its size and footprint due to financial constraints, hence we want to build the smallest accelerator possible which meets the clinical requirements and has normal conducting magnets. In order to assess the amount of bending required to first order (without considering the energy range), for a normal conducting strength magnet arrangement we may calculate the total length of the bending $L_{\text {bend }}$ for the maximum rigidity beam $B \rho$ of $2.94 \mathrm{Tm}$ at $350 \mathrm{MeV}$, given the maximum magnetic field seen by a particle on the closed orbit has a constraint $B_{0, \max }<1.8 \mathrm{~T}$ and divide this by the number of cells in the lattice $N_{\text {cells }}$,

$$
L_{\text {bend }}=\frac{1}{N_{\text {cells }}} \frac{2 \pi B \rho_{\max }}{B_{0, \max }} .
$$

The design will use a scaling FFAG magnet type, where the radial field profile is given by

$$
B(r)=B_{0}\left(\frac{r}{r_{0}}\right)^{k},
$$

where $B_{0}$ is the magnetic field at the reference radius $r_{0}$ and $k$ is the field index. The packing factor of the cell is then given by 


$$
\alpha=\frac{L_{\text {trip }}}{L_{\text {cell }}}
$$

where the length of the cell and the triplet are given, respectively, by

$$
\begin{gathered}
L_{\text {cell }}=2 L_{L D}+3 L_{M}+2 L_{S D} \\
L_{\text {trip }}=3 L_{M}+2 L_{S D}
\end{gathered}
$$

and the ratio of the short drift $L_{S D}$ to the magnet length $L_{M}$ is $R_{S M}=L_{S D} / L_{M}$. A typical FDF triplet arrangement was selected for the cell which allows focusing of a compact beam, limiting the aperture of the magnets and beam pipe $[43,44]$. The length of the focusing $\left(L_{F}\right)$ and defocusing $\left(L_{D}\right)$ magnets (where $\left.L_{F}=L_{D}\right)$ is given by $L_{\text {bend. }}$.

Figure 1 shows the geometric parameters of the FDF cell which were used to optimize the size of the ring lattice. The lengths of the FFAG sector magnets and intermediate drift spaces indicated in Fig. 1 represent the respective component lengths at the radius $r_{0}$. Large, normal conducting magnets for scaling FFAGs have already been demonstrated with dimensions of approximately $1.0 \mathrm{~m}$ in length and width $[26,27,45]$. In order to ensure that our magnet design is realistic, the limit on the magnet length of $\sim 1.0 \mathrm{~m}$ is suggested, and the magnet aperture to $\sim 0.5 \mathrm{~m}$ in order to reduce the horizontal orbit excursion over the energy range.

An Enge-type fringe field with a fixed extent $(\sim 10 \mathrm{~cm})$ was used throughout this work to simulate the field fall-off at the end of the magnets [46]. The overlap of fields between the magnets is modeled by superposition. For future studies we intend to use 3D magnet modeling to provide more realistic fringe fields. If this is found to reduce the tune flatness, methods of mitigation such as field clamps will be considered.

The number of cells in the lattice is selected by assessing the relative size of the magnets, the peak magnetic field constraint $B_{0, \max }<1.8 \mathrm{~T}$ (the maximum magnetic field seen by a particle on the highest energy closed orbit) to prevent saturation of the magnetic field [40] and the average horizontal orbit excursion around the ring over

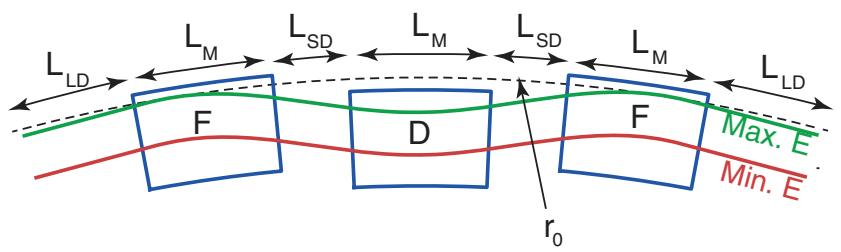

FIG. 1. Geometry of the FDF triplet cell used in NORMA showing the sector-type magnets in blue, the minimum and maximum energy orbits ( 30 and $350 \mathrm{MeV}$ ) in red and green, respectively, and the reference radius $r_{0}$ as a dashed black line. For optimizing the geometry, the free parameters were selected as the cell length $L_{\text {cell }}=2 L_{L D}+3 L_{M}+2 L_{S D}$, the triplet length $L_{\text {trip }}=3 L_{M}+2 L_{S D}$, and the packing factor $\alpha=L_{\text {trip }} / L_{\text {cell }}$. the energy range given some target lattice radius. The average horizontal orbit excursion around the ring over the energy range is used in this study as the maximum in the $\mathrm{F}$ magnet is typically only around $1 \mathrm{~cm}$ larger than the average. If we fix the radius and $B_{0, \max }$, then a higher number of cells requires more tightly packed magnetshence the focusing must be stronger to maintain stability. A possible solution to this is increasing $B_{0, \max }$, however we wish to keep this $<1.8 \mathrm{~T}$. The lower the number of cells, the greater the magnet spacing must be for a fixed magnet size, thus reducing $B_{0, \max }$ in order to maintain stability. The lower the magnetic field, the larger the horizontal orbit excursion over a given energy range which is undesirable due to the larger aperture magnets needed which can be difficult and costly to construct.

Previous studies [30] showed the relationship between number of cells, field index and maximum magnetic field strength in a proton scaling FFAG and upon selecting a maximum field strength, calculated various geometric parameters. As we are exploring a proton scaling FFAG in a similar kinetic energy regime, we follow a similar method for a fixed radius of $10 \mathrm{~m}$, fixed ring tune of 8.7 and 3.5 in the horizontal and vertical planes, respectively (similar to the PAMELA lattice) and the higher maximum kinetic energy of $350 \mathrm{MeV}$ but restrict $B_{0, \max }<1.8 \mathrm{~T}$. Table II shows some of the parameters in this fixed radius study; lattices with $>14$ cells were unstable at the fixed working point due to the tight packing of the magnets and the high magnetic field required for focusing. The 12-cell lattice has a small average horizontal orbit excursion and a magnet length $<1.0 \mathrm{~m}$. However, a previous study was carried out with the 12-cell lattice [47] where it could be noted that although a stable optimized solution could be found, the DA for 1000 machine turns (approximately that required for acceleration over the energy range) did not remain above $40 \mathrm{~mm}$ mrad when magnet misalignment errors were considered and a racetrack proved hard to

TABLE II. The peak magnetic field $B_{0, \max }$ in the $\mathrm{F}$ and $\mathrm{D}$ magnets, magnet length (same for $\mathrm{F}$ and $\mathrm{D}$ ) and the average horizontal orbit excursion around the ring over the energy range for a fixed radius, fixed working point lattice with a variable, even number of cells. Lattices with $<8$ and $>14$ cells were unstable in this fixed working point study, however stability could be realized at a different working point.

\begin{tabular}{lcccc}
\hline \hline & $B_{0, \max }$ & $\begin{array}{c}B_{0, \max } \\
\text { No. of cells }\end{array}$ & $\begin{array}{c}\text { Magnet } \\
\text { in F [T] }\end{array}$ & $\begin{array}{c}\text { Orbit } \\
\text { in D [T] }\end{array}$ \\
\hline 6 & N/A & N/A & N/A & N/A \\
8 & 1.47 & -1.07 & 1.28 & 0.69 \\
10 & 1.58 & -1.22 & 1.03 & 0.46 \\
12 & 1.64 & -1.31 & 0.86 & 0.33 \\
14 & 1.70 & -1.39 & 0.73 & 0.25 \\
16 & N/A & N/A & N/A & N/A \\
\hline \hline
\end{tabular}


optimize. The lattice with 10 cells fits our requirements, and was hence selected for the study presented in this paper.

\section{B. Lattice optimization in Pyzgoubi}

In this section we describe the methods we have developed in pyzgoubi which allow the optimization of a lattice over a wide range of parameters [41]. This powerful feature in Pyzgoubi allows us to define a range of desired lattice criteria such as working point, closed orbit range, Courant-Snyder parameters for matching and so forth, and a set of free parameters which the optimizer can vary over the configuration space to reach the desired outcome. Pyzgoubi allows the user to take advantage of external SciPy routines [48] to optimize these parameters.

In a typical optimization routine an input vector of parameters is defined, for example magnet strengths and gradients. In each iteration of the optimization a lattice is constructed using the current input vector, the lattice parameters are calculated using the get_cell_ properties () function in Pyzgoubi [41] and a penalty function is created which consists of several user-defined contributions. These could be for example, the distance to the target tune or the size of the horizontal orbit excursion over the energy range. A SciPy routine such as downhill simplex [49] is then used to minimize the penalty function within a given tolerance. The flexibility of the optimization capability in Pyzgoubi should be noted; the user may define any number of constraints and free parameters they wish to enable them to realize the desired lattice. We now demonstrate the use of a specific optimization procedure in the NORMA design.

\section{Reducing circumference for a fixed working point}

To calculate the optimum circumference of the ring lattice given the cell geometry in Fig. 1, we initially fix the working point and adjust the drift lengths $L_{S D}$ and $L_{L D}$. By fixing $L_{M}$ as a constant and allowing the packing factor $\alpha$ and the ratio $R_{S M}$ to vary as free parameters, we used our optimization procedure to obtain the magnet parameters required for transverse stability and fixed working point for a given $L_{S D}$ and $L_{L D}$.

The initial working point was fixed at ring tune $Q_{h}=7.7, Q_{v}=2.7$ in the horizontal and vertical planes, respectively as this is away from resonances in the second stability region within a region of high DA (see Sec. II B 2 for information about the method of determining a high DA region). The strengths of the $\mathrm{F}$ and $\mathrm{D}$ magnets at $r_{0}\left(B_{0, F}\right.$ and $B_{0, D}$ ) and the field index $k$ (common to both magnets) were defined as the input vector of free parameters in the optimization procedure; this allows the tune and stability of a given lattice to be maintained.

The optimization penalty function had the major contributions: a closed orbit with $|\operatorname{Tr}(M)|<2$ must exist at all energies over the energy range; machine radius is controlled by minimizing the distance of the highest energy orbit to $r_{0}$; distance to the fixed working point is minimized; field strengths and index must be in the second stability region of Hill's equation.

Figure 2 shows the circumference and long drift length $L_{L D}$ for stable lattices over the energy range in the parameter space optimized in Pyzgoubi when $\alpha$ and $R_{S M}$ are varied. Lattices which were within \pm 0.1 of the fixed working point were still plotted if they were calculated to be stable in order to give a visual representation for a wide range of circumference, $\alpha$ and $R_{S M}$. The PAMELA design specified around $1.7 \mathrm{~m}$ between magnets which allowed space for the cryogenic modules, rf cavities, and other diagnostic equipment. Although we do not include cryogenically cooled magnets in our design, we still need to have sufficient space for the magnet end coils and associated shielding plates required on such large normal conducting magnets, and to allow space for rf modules and diagnostic equipment. To achieve this we specified $2.0 \mathrm{~m}$ of magnetfree drift between magnets in neighboring cells.

The PAMELA collaboration showed that a DA above about $40-50 \mathrm{~mm}$ mrad was sufficient for a medical accelerator [31]. Similarly, we require our normalized DA over 1000 machine turns (approximately the number needed for acceleration over the energy range) to be above $40 \mathrm{~mm} \mathrm{mrad}$ in order that injected bunches may be efficiently captured (see Sec. IV).

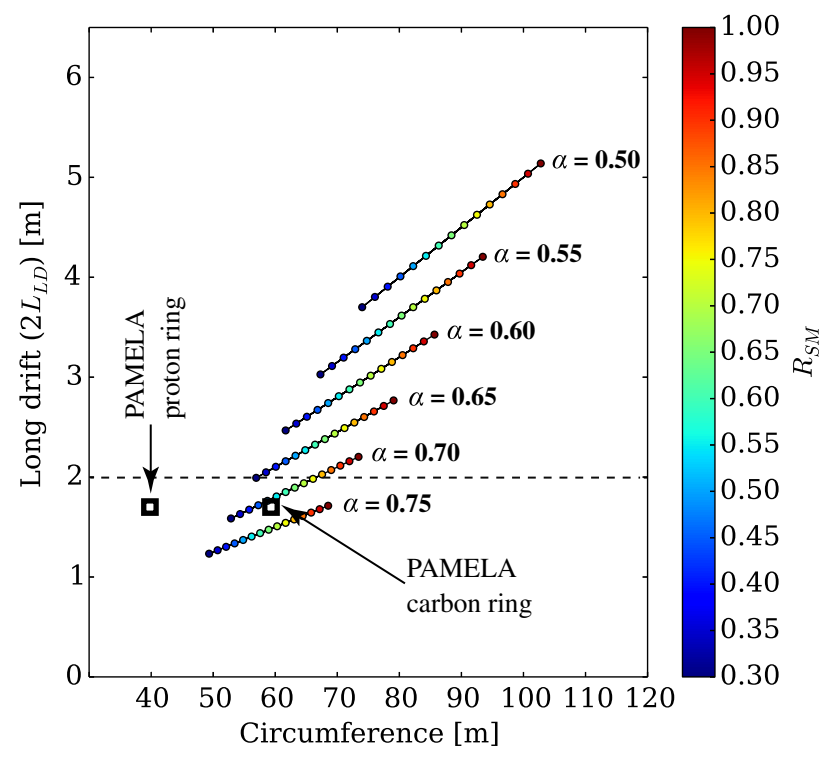

FIG. 2. Stable 10 cell ring lattices using optimization in Pyzgoubi in the geometric parameter space. The color of each point indicates the ratio $R_{S M}$. The location of the PAMELA proton and carbon rings are shown in the parameter space for comparison. The dashed line at $2 L_{L D}=2.0 \mathrm{~m}$ indicates our requirement limit for the long drift between magnets in neighboring cells. The lattice becomes unstable when increasing $\alpha$ above 0.75 and reducing $R_{S M}$ below 0.3 . at the fixed working point at $Q_{h}=7.7, Q_{v}=2.7$. 
The DA at the fixed working point was calculated for the geometric parameter space shown in Fig. 2 by tracking 16 combinations of particles for 1000 machine turns with different coordinates in the horizontal and vertical phase space, given by using the initial Courant-Snyder parameters [50]. The minimum of the 16 combinations is selected to give a robust figure for the dynamical acceptance of a linearly matched elliptical bunch at injection. This method assumes that a linearly matched, elliptical bunch from an injector will be easier to match into a FFAG than a more complicated, nonlinearly matched bunch. It should be noted that such a DA measurement is usually lower than conventional measurements carried out using the largest invariant trajectory in phase space, however our measurement may be more realistic when considering a real injected bunch.

Figure 3 shows the DA in the parameter space defined by $\alpha$ and $R_{S M}$. However, in this plot the tune is constrained in the optimizer to within 0.001 of the target at $Q_{h}=7.7$, $Q_{v}=2.7$ in order that the DA can be sensibly compared between geometries, hence Fig. 3 allows one to observe the effect on the DA for different packing arrangements in the lattice at a fixed tune point. Generally, the larger the lattice and the more space between magnets, the higher the DA due to the weaker focusing required. Due to the tightly controlled tune point in Fig. 3, we observe less data for larger geometry rings because the tune point for the optimum DA changes for lattices outside this plot. However we are able to see the important relationship in Fig. 3 which allows us to make a good choice of geometry.

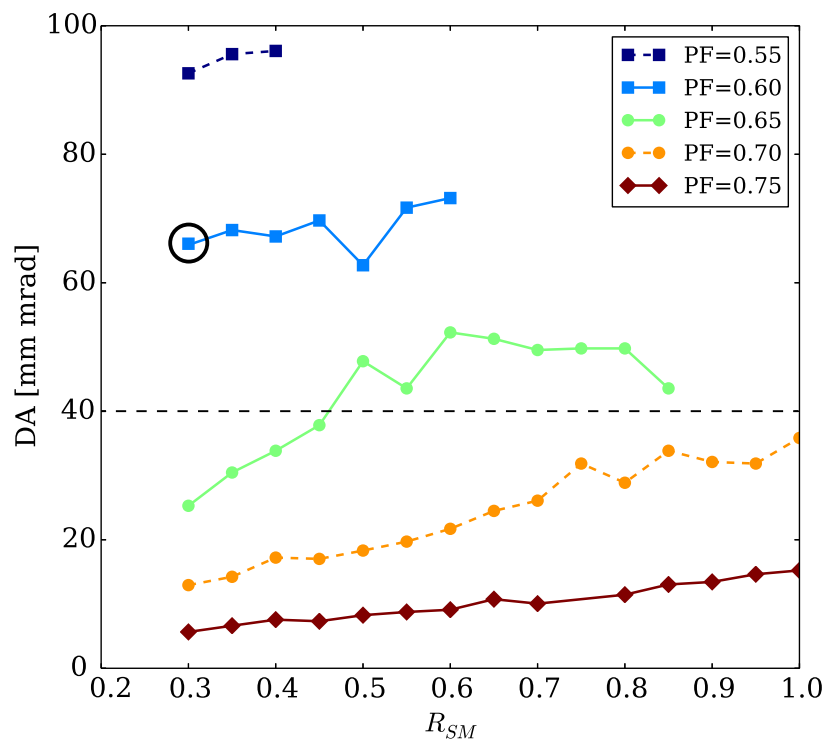

FIG. 3. The DA is shown for the stable lattices in Fig. 2 which have the required target tune at $Q_{h}=7.7, Q_{v}=2.7$. As $R_{S M}$ decreases and the packing factor ( $\mathrm{PF}$ in the figure key) increases, the lattice circumference reduces. The smallest circumference lattice with a DA above our threshold of $40 \mathrm{~mm} \mathrm{mrad}$, this lattice is indicated by a black circle.
By inspecting Figs. 2 and 3 we are able to calculate stable parameters for the smallest lattice which fits our desired criteria; $2 L_{L D} \geq 2.0 \mathrm{~m}$ and DA $>40 \mathrm{~mm} \mathrm{mrad}$ at a given working point. Hence the optimized lattice has $\alpha=0.6$ and $R_{S M}=0.3$ where the average radius is $9.61 \mathrm{~m}$. As this lattice comprises scaling FFAG sector magnets, the geometry of the full lattice can be expressed by the angle $A_{i}$ of each element which corresponds to the length $L_{i}$ at $r_{0}$ given in Fig. 1. The angles $A_{L D}, A_{S D}$ and $A_{M}$ are, respectively, 7.2, 1.8 and 6.0 degrees.

\section{Optimizing the working point and dynamic aperture for a fixed geometry}

Having chosen a suitable fixed geometry we now present our procedure for optimizing the working point in Pyzgoubi so as to select a suitable region away from potential resonances and to maximize the DA in an error-free, ideal lattice. When computing the DA over a large parameter space, we used computationally intense methods which yield a very high level of precision and allow us to make a highly precise parameter selection. We used the University of Manchester's High Throughput Computing (HTC) Condor cluster [51] in order to perform tens of thousands of parallel calculations, each of which individually take approximately $10 \mathrm{~h}$ to complete. For example, we developed algorithms which used the Condor system to calculate and recombine data over an area of tune space with $>1 \times 10^{4}$ individual DA calculations in approximately a $24 \mathrm{~h}$ period, allowing rapid, highly precise parameter exploration. Without HTC calculations of this precision such processes could have taken $\sim$ years to complete. Such high resolution calculations enable one to see fine structure in the tune space such as the location of instabilities and resonances, and allows one to avoid such regions.

Figure 4 shows the parameter space of the second stability region of Hill's equation where the cell tunes are indicated. The separation between the first and second stability regions can clearly be seen, separated by the half integer cell tune. Pyzgoubi was used to calculate the DA in the second stability region of this parameter space. Figure 5 shows the DA as a function of the cell tune, where a strong sextupole resonance at horizontal cell tune 0.66 means the DA has a very small finite value until around 0.73 onward, hence the relevant area is shown. The highest DA region is at a ring tune of $Q_{h}=7.723$ and $Q_{v}=2.735$ which is away from resonances and where the DA is $>60.0 \mathrm{~mm}$ mrad. Computing time limits the resolution of the DA in the tune space, as in Fig. 5; however once a target area for the working point is selected, it is possible to increase the accuracy of the optimization by locally inspecting the DA in the parameter space with a higher resolution. The DA in the parameter space is shown in detail local to the working point $\left(Q_{h}=7.723, Q_{v}=2.735\right)$ in Fig. 6, where the original optimized value and the highest optimized value are highlighted showing the 


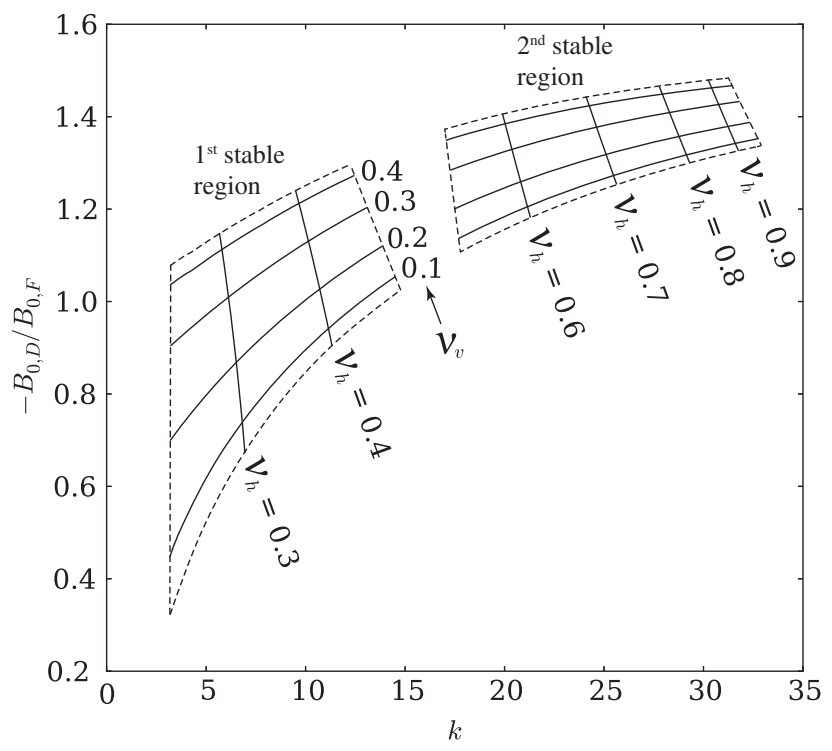

FIG. 4. Parameter space which was studied for the NORMA ring lattice. The region encapsulating stable lattices is shown by a dashed area and the horizontal $\nu_{h}$ and vertical $\nu_{v}$ cell tunes are shown with solid lines. The first and second stability region of Hill's equation are indicated; separated by the half-integer cell tune.

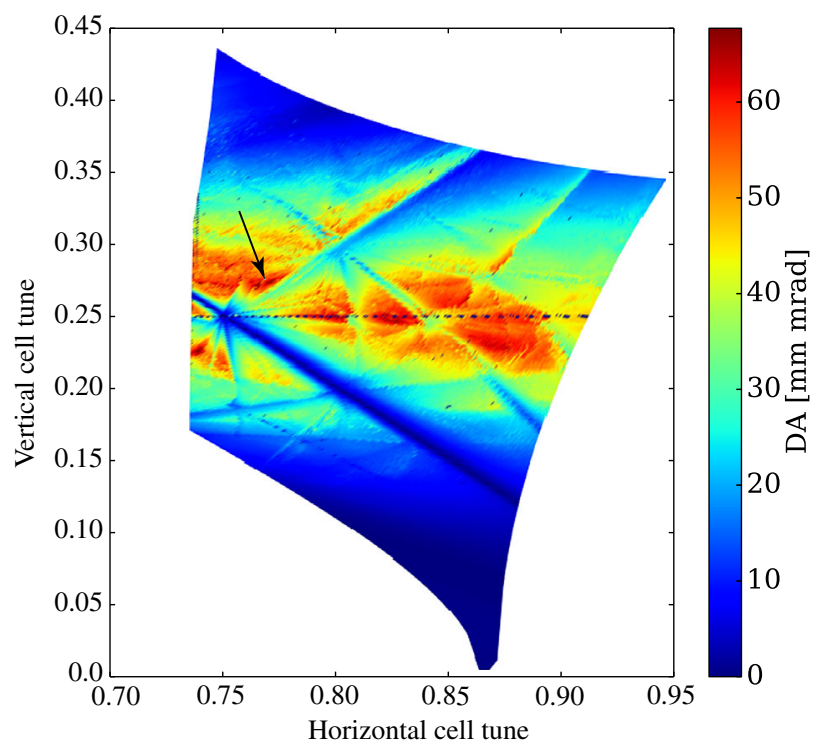

FIG. 5. The DA is shown in the tune space for an error free NORMA ring lattice. The DA is represented by a color spectrum with higher DA appearing in red and lower DA toward blue. The darkest blue points represent dynamically unstable lattices, for example those along the systematic resonance at vertical tune of 0.25 . The black arrow indicates the region containing the highest DA. Due to the strong sextupole resonance at horizontal cell tune 0.66 , the DA is only shown from around 0.73 onward. Over $1 \times 10^{4}$ individual calculations exist within this plot, allowing high resolution analysis.

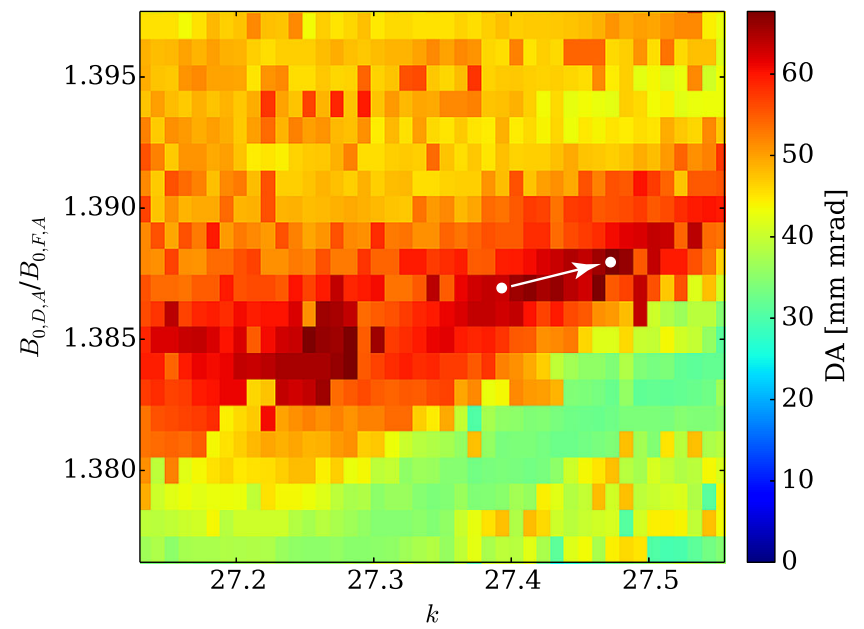

FIG. 6. The DA in the parameter space around the highest optimized value (darker red). The white points and arrow show how the original lattice found by the optimizer is changed (direction of the arrow) to a point in the parameter space with a higher DA.

direction of the optimization. The final optimized DA is $68 \mathrm{~mm} \mathrm{mrad}$ and the main parameters of this lattice are given in Table III. This shows we are able to optimize a ring lattice in Pyzgoubi for protons up to $350 \mathrm{MeV}$ which has an average radius of $9.61 \mathrm{~m}$ an average horizontal orbit excursion of $43 \mathrm{~cm}$ (maximum $44 \mathrm{~cm}$ in the centre of the $\mathrm{F}$ magnet) and a DA of $68 \mathrm{~mm} \mathrm{mrad}$.

\section{Properties of the optimized NORMA ring lattice}

In this section we present the properties of the optimized NORMA ring lattice, the main parameters of which were given in Table III. A schematic of this lattice is shown in Fig. 7 where the approximate size and shape of the magnets are shown, and the position of the minimum and maximum orbits are indicated. The drift space between F magnets in

TABLE III. The main parameters of the optimized NORMA lattice.

\begin{tabular}{lc}
\hline \hline Parameter [unit] & Value \\
\hline Average radius [m] & 9.61 \\
Circumference [m] & 60.4 \\
Average horizontal orbit excursion [m] & 0.43 \\
Ring tune $\left(Q_{h}, Q_{v}\right)$ & $7.72,2.74$ \\
$k$ & 27.47 \\
Max. field seen by beam in F magnet [T] & 1.57 \\
Max. field seen by beam in D magnet [T] & -1.19 \\
DA [mm mrad] & 68.0 \\
Magnet-free drift $2 L_{L D}[\mathrm{~m}]$ & 2.4 \\
$A_{L D}$ [deg.] & 7.2 \\
$A_{S D}$ [deg.] & 1.8 \\
$A_{M}$ [deg.] & 6.0 \\
\hline \hline
\end{tabular}




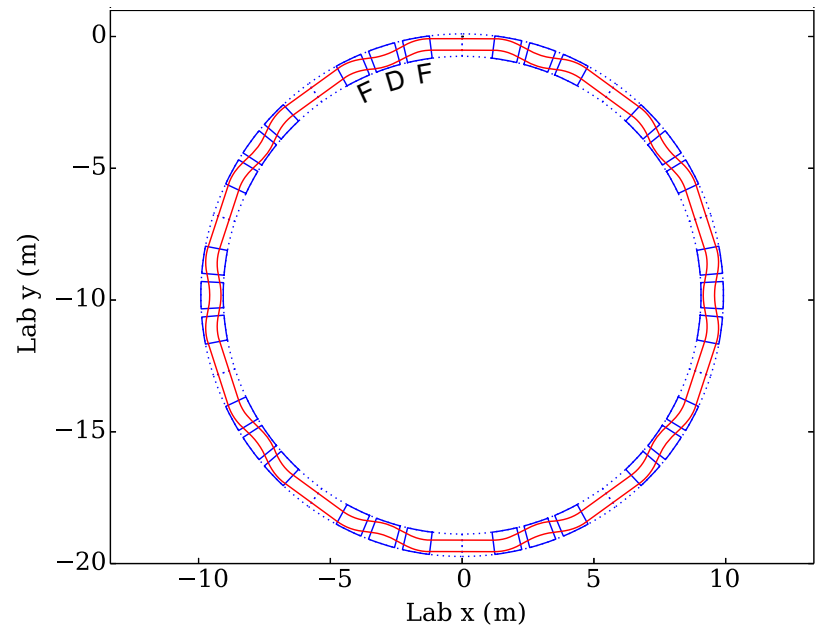

FIG. 7. A schematic of the NORMA ring lattice showing the lowest (inner) and highest (outer) energy orbits in red. Magnets are outlined by solid blue lines and the cell boundaries as dashed blue lines. The approximate positions of the F and D magnets are indicated for one cell. Note that the solid blue outlines indicate only the approximate radial positions and horizontal aperture of the magnets, however the sector width of each is accurate.

neighboring cells is $\sim 2.4 \mathrm{~m}$ which is sufficient for $\mathrm{rf}$ modules and diagnostics (see Sec. IV).

The change in tune between a particle on the closed orbit at minimum and maximum energy is $6.5 \times 10^{-5}$ and $9.1 \times$ $10^{-4}$ for horizontal and vertical tune, respectively. The flatness of the tune over the energy range in our initial Pyzgoubi model is due to the ideal scaling of the FFAG magnets and allows harmful resonances to be avoided given our working point selection. However, in a real magnet the varying gap size required to create a scaling FFAG magnetic field leads to a change in the flutter and hence a variation in the vertical tune over the energy range; this will be studied in due course with a realistic magnet design.

The horizontal and vertical $\beta$-functions and the dispersion at the injection energy of $30 \mathrm{MeV}$ are shown in Fig. 8.

\section{Cell misalignment errors}

Magnet misalignment errors were simulated in Pyzgoubi by transversely offsetting each of the 10 cells randomly using a Gaussian distribution with a standard deviation of $\sigma$ and a cutoff at $3 \sigma$. Both horizontal and vertical cell misalignments were implemented and 50 different randomly offset lattices were created for each value of $\sigma$. The DA for each error lattice was measured for the injection energy of $30 \mathrm{MeV}$. The effect on the DA for the misalignments can be seen in Fig. 9 where the DA remains above $40 \mathrm{~mm} \mathrm{mrad}$ with up to $100 \mu \mathrm{m}$ transverse magnet misalignments. The gradient of the best-fit line in Fig. 9 is $74.3 \mathrm{mrad}$ which we call the DA reduction factor; this

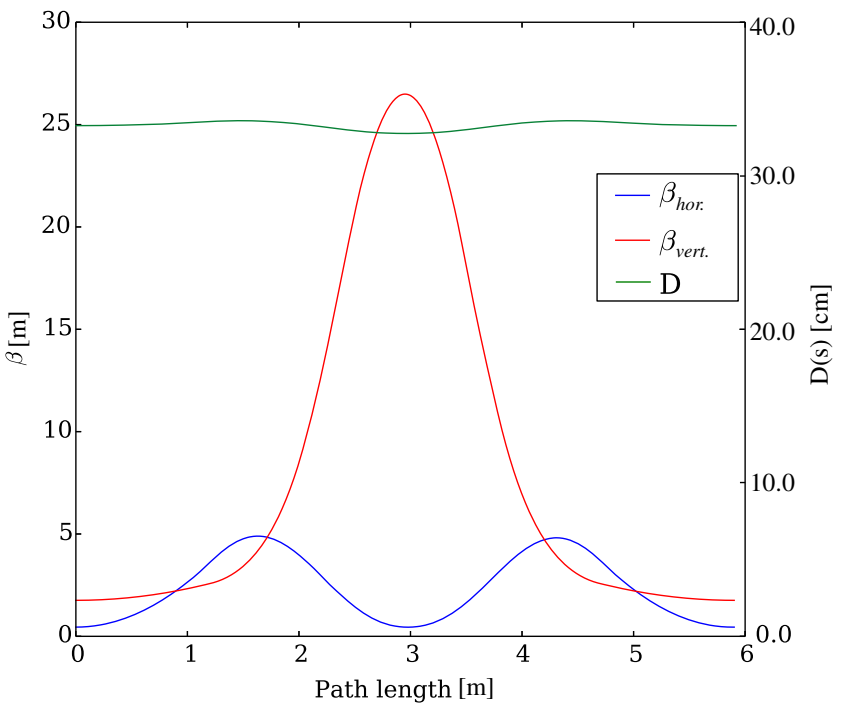

FIG. 8. The horizontal and vertical $\beta$-functions and the dispersion in the cell for the NORMA ring lattice are shown at the injection energy of $30 \mathrm{MeV}$.

will later be compared to the racetrack configuration in Sec. III. The unaccelerated horizontal and vertical rms closed orbit distortion (COD) at the injection energy of $30 \mathrm{MeV}$ can be seen in Fig. 10. The minimum chi-squared fits indicated by the red lines in Figs. 10(a) and (b) show the average increase in the COD as a function of $\sigma$; this is known as the amplification factor. The amplification factors are 3.0 and 3.5, respectively, for the horizontal and vertical

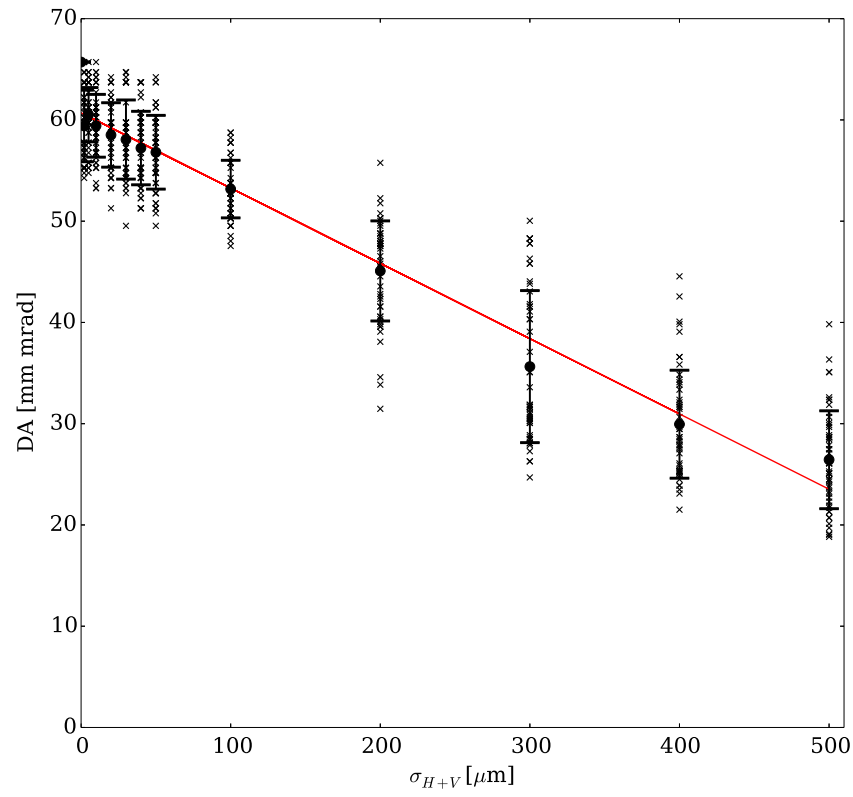

FIG. 9. The DA as a function of horizontal and vertical cell misalignments with a Gaussian distribution of width $\sigma$. Each individual randomly offset lattice is shown as a cross, the mean is shown as a filled circle, and the standard deviation as the error bars. The red line shows a minimum chi-squared fit to the data. 


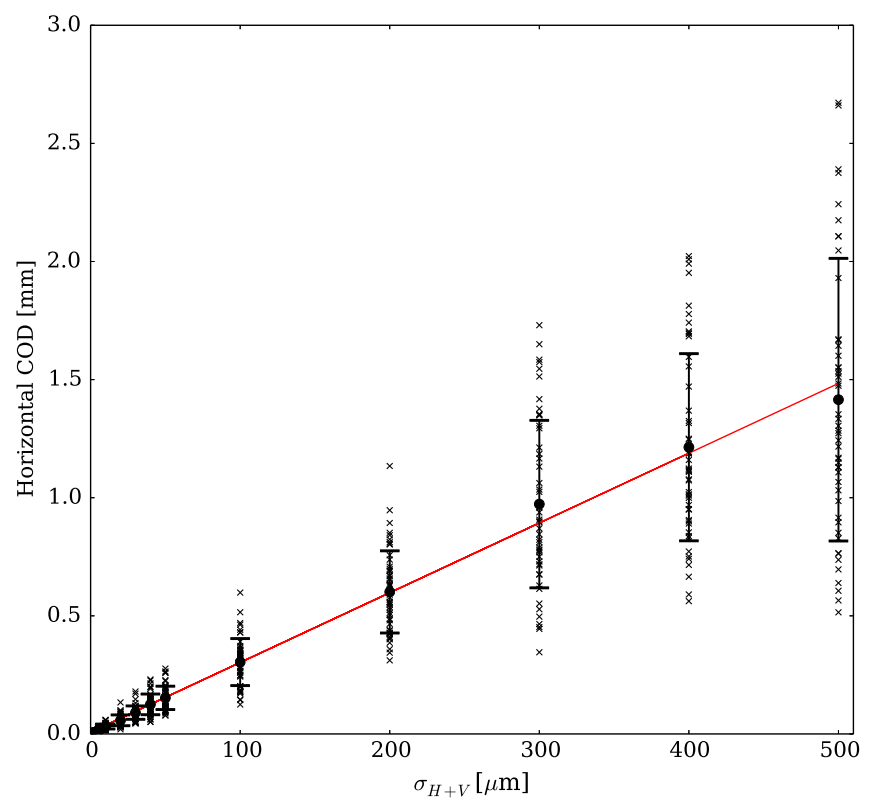

(a)

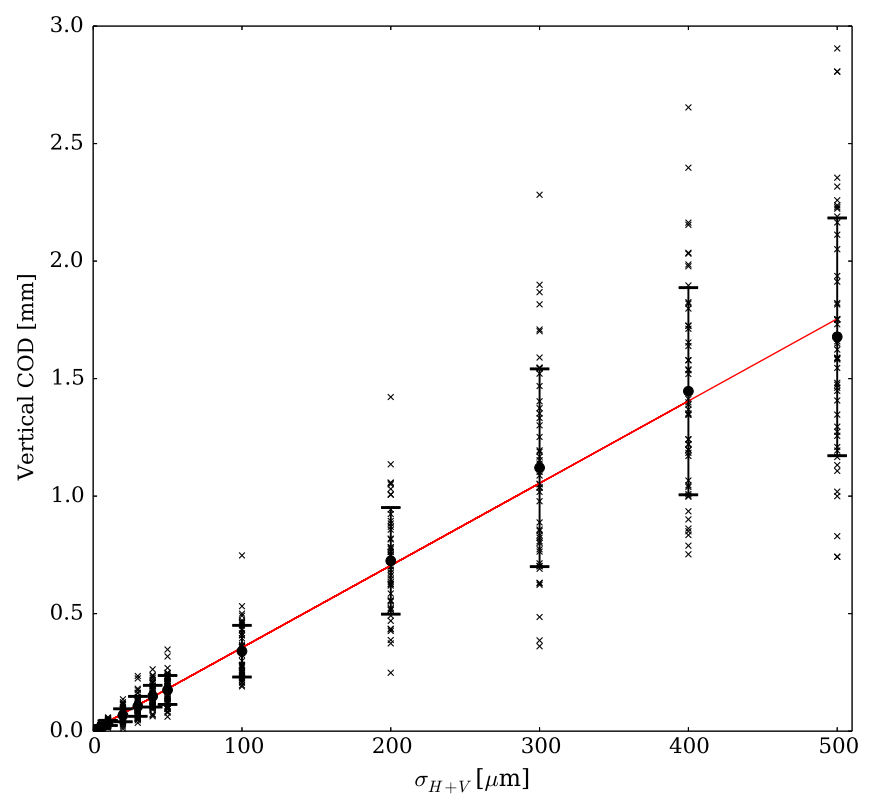

(b)

FIG. 10. The horizontal (a) and vertical (b) rms COD with cell misalignment errors. Each individual randomly offset lattice is shown as a cross, the mean is shown as a filled circle, and the standard deviation as the error bars. The red line shows a minimum chi-squared fit to the data.

COD. The PAMELA collaboration calculated the mean amplification factor of accelerated orbits to be 5.81 and 9.47 in the horizontal and vertical directions, respectively, and asserted that for an allowed orbit distortion of $\approx 1 \mathrm{~mm}$, the alignment tolerances must be better than around $100 \mu \mathrm{m}$, which is routinely achievable in other accelerators [31].

The COD in both planes for NORMA in Fig. 10 remains $<0.5 \mathrm{~mm}$ with $100 \mu \mathrm{m}$ misalignments, where $\approx 100 \mu \mathrm{m}$ is considered practically realizable and could be controlled by corrector magnets. The rms tune variation between the ideal and error lattices with $\sigma=100 \mu \mathrm{m}$ at injection energy is $3.9 \times 10^{-5}$ and $3.7 \times 10^{-5}$ for the horizontal and vertical tunes, respectively, allowing the DA to remain above $40 \mathrm{~mm} \mathrm{mrad}$ in this case.

\section{Concluding remarks-NORMA ring lattice}

We have used Pyzgoubi to optimize the design for a 30 $350 \mathrm{MeV}$, normal conducting, proton scaling FFAG ring lattice capable of $\mathrm{pCT}$ in a clinical environment. The lattice consists of 10 cells, has an average radius of $9.61 \mathrm{~m}$, an average horizontal orbit excursion over the energy range of $43 \mathrm{~cm}$, a maximum orbit excursion in the $\mathrm{F}$ magnet of $44 \mathrm{~cm}$ and a DA of $68 \mathrm{~mm}$ mrad. The tolerance to magnet alignment errors allows the DA to remain above $40 \mathrm{~mm}$ mrad with a distribution of misalignments with $\sigma=100 \mu \mathrm{m}$. The magnets are $1.0 \mathrm{~m}$ long with $\sim 0.5 \mathrm{~m}$ horizontal aperture. The peak magnetic field seen by the beam $B_{0, \max }$ in the $\mathrm{F}$ and $\mathrm{D}$ magnets is within the normal conducting range at $1.57 \mathrm{~T}$ and $-1.19 \mathrm{~T}$, respectively.

There is approximately $2.0 \mathrm{~m}$ of magnet-free straight between cells, in which some rf modules and diagnostic equipment may be placed as well as magnet field clamps, etc. However, in order to facilitate injection and extraction systems, we shall now explore modifying the NORMA ring lattice to form a racetrack.

\section{THE NORMA RACETRACK LATTICE}

Previous studies showed that it was possible to break the symmetry of a conventional circular FFAG by incorporating a number of long straight, magnet-free insertions at opposing points in the ring [52]. In this section we show a racetrack configuration derived from the NORMA ring lattice by breaking the symmetry and inserting magnet-free drift spaces at two opposite points in the ring. In order to do this, the lattice definition is modified to include a matching cell and the procedure used to optimize the ring lattice is modified. In this section we first outline the procedure used to create stable racetrack configurations from the ring with a variable amount of extra drift space at two points. We then show the effect on the DA of increasing this drift length and we select a particular racetrack solution to study in detail.

The lattice definition was modified for the purposes of creating a racetrack in order to match the optics into the longer straight sections. Instead of 10 identical FDF cells, the racetrack comprises six identical "arc" FDF cells and four "matching" FDF cells each side of the two longer drifts. Hence two unique cell definitions are created and we refer to them specifically as the arc cell and matching cell in this study. Figure 11 shows a schematic of the cell and magnet definitions and their locations in the racetrack. All the geometric cell parameters are kept constant with respect to the NORMA ring design; the only difference is the 


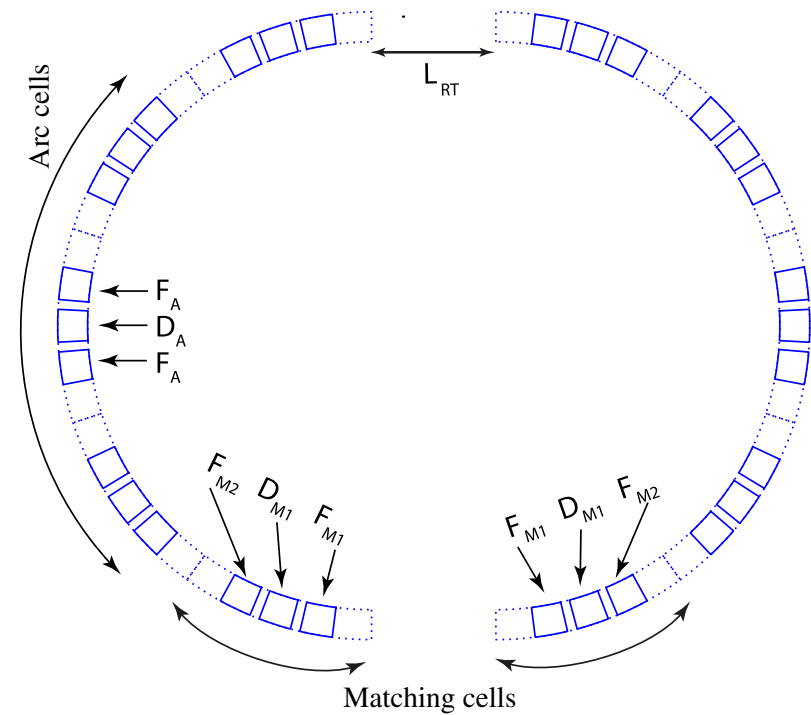

FIG. 11. Schematic of the racetrack cells showing the long magnet-free straights $L_{R T}$ and the two types of cell: arc and matching. Five families of magnets are shown, all have the same field index $k$ and together these make up the six free parameters used in the optimization. All of the six arc cells have just one type of $\mathrm{F}$ and one type of $\mathrm{D}$ magnet.

additional racetrack drift length $L_{R T}$, which is inserted at the symmetry points indicated in Fig. 11. In order to use Pyzgoubi to find a stable optimized and matched solution to this racetrack we define six free magnetic parameters: (i) $k$, the common field index between all magnet families, (ii) $B_{0, F, M 1}$, the field at $r_{0}$ in the $\mathrm{F}$ magnet immediately adjacent to the racetrack straight $L_{R T}$ in the matching cell, (iii) $B_{0, D, M 1}$, the field at $r_{0}$ in the $\mathrm{D}$ magnet in the middle of the matching cell, (iv) $B_{0, F, M 2}$, the field at $r_{0}$ in the $\mathrm{F}$ magnet which borders the three arc cells, (v) $B_{0, F, A}$, the field at $r_{0}$ in all the $\mathrm{F}$ magnets of the arc cells, (vi) $B_{0, D, A}$, the field at $r_{0}$ in all the $\mathrm{D}$ magnets of the arc cells. These parameters were used as the input vector for the racetrack optimization and were derived from the NORMA ring lattice, where the matching cell FDF magnets were given starting values equal to those of the arcs. Most of the criteria which contributed to the penalty function in the downhill simplex routine were the same as for the ring (see Sec. II B); crucially we kept the working point fixed to the ring tune in Table III during the optimization of the racetrack.

Due to the breaking of the ring lattice symmetry to make a racetrack, we must match the optics into the longer straight sections by using the matching cells. While the $\beta$ functions will necessarily change with respect to the ring during the matching, we nevertheless wish to restrict large changes in the $\beta$-functions resulting from potential optimization to local minima which could reduce the DA. In order to control the size of the $\beta$-functions we added four additional optimization criteria to the procedure in order to

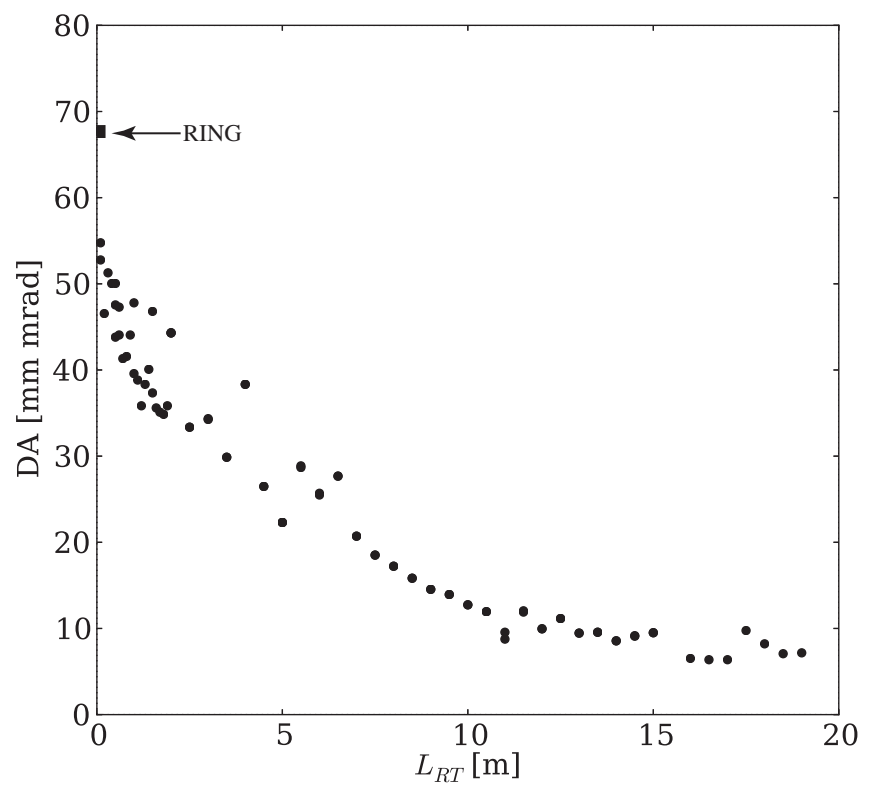

FIG. 12. The DA for racetracks as a function of $L_{R T}$ is shown. The DA for the NORMA ring lattice can be seen at $L_{R T}=0$.

restrict the maximum height and the minimum of the waist of each $\beta$-function.

The additional magnet-free straight $L_{R T}$ was increased from 0.0 to $20.0 \mathrm{~m}$ in serialized steps using the previous set of magnetic parameters as the initial input vector for the next iteration. The DA as a function of $L_{R T}$ can be seen in Fig. 12. After the symmetry of the ring lattice is broken, we can see that the DA drops with $L_{R T}$. The variation of the points is due to noise in the optimizer as the algorithm is sensitive to the initial parameters and local minima. However, we show in the next section that it is possible to optimize the DA of a given optimized lattice further, starting from an initial set of parameters such as any of those found by the optimizer in Fig. 12. The geometry of the cells in each racetrack is fixed except for $L_{R T}$ which means that in order to match into the long straight sections the particles must be relatively strongly focused in the final F magnet, $F_{M 1}$ before drifting into the long straight section. Figure 13 shows how the maximum field seen by a particle on the highest energy orbit changes as a function of $L_{R T}$ in the strongest magnet $F_{M 1}$. Whilst the fields seen by the highest energy orbit particle in the other magnets stay within the normal conducting range, the field seen by the highest energy orbit particle in the $F_{M 1}$ magnet increases into the superconducting region above $1.8 \mathrm{~T}$. Hence the racetracks with $L_{R T} \gtrsim 1.2 \mathrm{~m}$ require a particle with $350 \mathrm{MeV}$ to experience a field in the $F_{M 1}$ magnet $>1.8 \mathrm{~T}$. In order to keep the field in all the magnets $<1.8 \mathrm{~T}$, we may scale the magnet reference radius $r_{0}$ by a factor $1 / f_{R T}$ and each magnet strength by $f_{R T}$ where $0<f_{R T}<1$. This allows the geometry of the cells and the optics to scale up and the magnetic field strengths to scale down appropriately. The length $L_{R T}$ must also be scaled up 


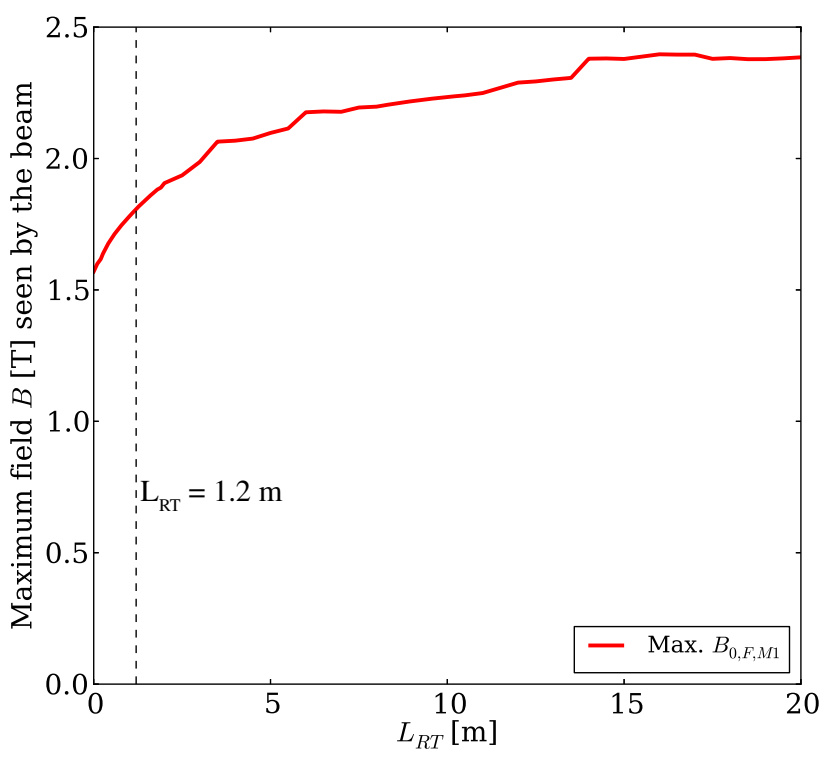

FIG. 13. The field seen by the highest energy orbit particle for the strongest magnet $\left(F_{M 1}\right)$ in the matching cells before the long straight sections. The field increases into the superconducting region above $1.8 \mathrm{~T}$ after around $1.2 \mathrm{~m}$ of magnet-free straight is added to the symmetric ring.

by $1 / f_{R T}$ in order to match the optics and retain the same tunes as the initial optimized racetrack.

As an example, in the next section we select a racetrack with $L_{R T}=2.0 \mathrm{~m}$-sufficient for easy injection and extraction as the total magnet-free straight including $2 L_{L D}$ is $4.4 \mathrm{~m}$. The $F_{M 1}$ magnet in this lattice, as optimized and shown in Fig. 12, has a field such that a particle with $350 \mathrm{MeV}$ would see a $1.9 \mathrm{~T}$ field. We will demonstrate how this example lattice can be scaled up in size slightly in order to reduce the field below $1.8 \mathrm{~T}$.

\section{A. NORMA racetrack with $L_{R T}=\mathbf{2 . 0} \mathrm{m}$}

Using the racetrack optimization procedure we were able to realize a racetrack with $L_{R T}=2.0 \mathrm{~m}$ (see Fig. 12) which has long straight sections between $F_{M 1}$ magnets of $4.4 \mathrm{~m}$ and a DA above $40 \mathrm{~mm} \mathrm{mrad}$; the parameters are shown in Table IV in the "unscaled" column where $f_{R T}=1.0$. In order to reduce the field in $F_{M 1}$ magnet we apply the scaling factor $f_{R T}=0.91$; this results in the parameters in the "scaled" column in Table IV.

In order to find the optimized DA for this racetrack, the local parameter space around the region located by the optimizer was studied and is shown in Fig. 14. The magnetic parameters for this lattice are the same as the scaled parameters in Table IV apart from the value of $B_{0, D, A}$ which changes from -1.588 to -1.584 in order to increase the DA from 50.0 to $57.7 \mathrm{~mm}$ mrad. A schematic of this racetrack is shown in Fig. 15 where the long straight section of length $4.9 \mathrm{~m}$ is indicated. The ring tune change over the energy range is 0.02 and 0.0045 in the horizontal and
TABLE IV. The main parameters of the NORMA racetrack with $L_{R T}=2.0 \mathrm{~m}$ after initial optimization when $f_{R T}=1.0$, and after scaling when $f_{R T}=0.91$.

\begin{tabular}{lcc}
\hline \hline Parameter [unit] & $\begin{array}{c}f_{R T}=1.0 \\
\text { (unscaled) }\end{array}$ & $\begin{array}{c}f_{R T}=0.91 \\
\text { (scaled) }\end{array}$ \\
\hline Average radius $r_{0}[\mathrm{~m}]$ & 9.61 & 10.55 \\
Circumference [m] & 64.4 & 70.7 \\
Average horizontal & 0.44 & 0.49 \\
$\quad$ orbit excursion [m] & & \\
Average ring tune & $7.70,2.66$ & $7.71,2.68$ \\
$\quad\left(Q_{h}, Q_{v}\right)$ & & \\
$k$ & 26.4 & 26.4 \\
Peak $F_{M 1}$ field [T] & 1.91 & 1.74 \\
DA [mm mrad] & 52.0 & 57.7 \\
Magnet-free drift $[\mathrm{m}]$ & 4.4 & 4.9 \\
\hline \hline
\end{tabular}

vertical, respectively, which is larger than the ring but still small enough to keep the tune away from resonances. The $\beta$-functions are shown in Fig. 16.

Magnet misalignments were studied in the same way as for the ring. Figure 17 shows the DA reduction as a function of the error distribution rms value $\sigma$. The gradient of the best-fit line in Fig. 17 (the DA reduction factor) is $67.5 \mathrm{mrad}$. Similar to the ring design, the DA in the racetrack can be kept above $40.0 \mathrm{~mm}$ mrad with $\sigma_{H+V}$ up to $100 \mu \mathrm{m}$.

The horizontal and vertical COD as a function of $\sigma$ can be seen in Fig. 18(a) and (b) where the amplification factors are calculated, respectively, as 2.1 and 1.8; a summary of the comparison between the ring and racetrack DA reduction factor, COD amplification factors and rms tune

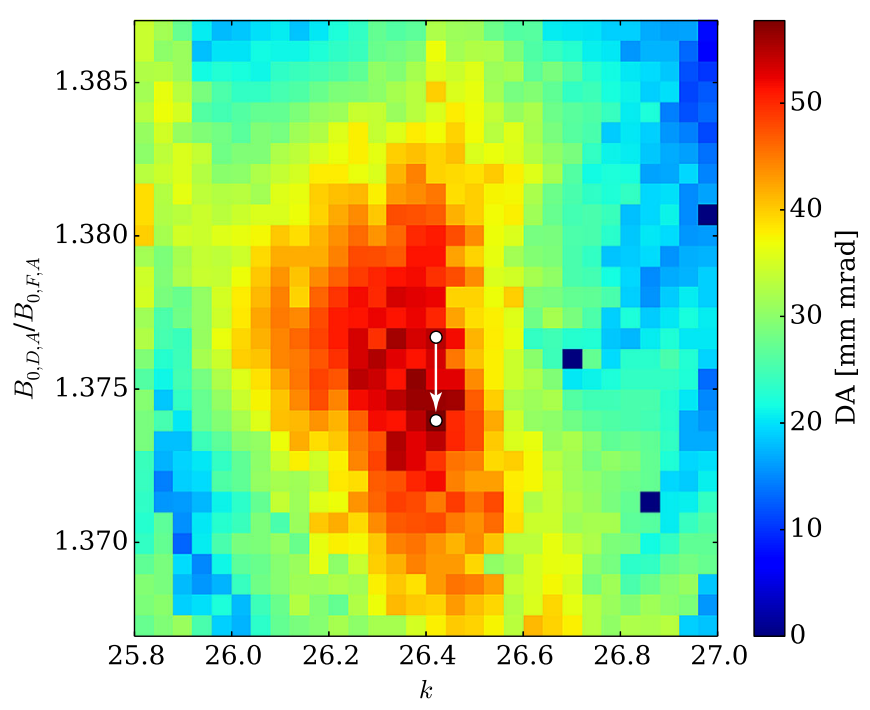

FIG. 14. The DA in the parameter space around the region found by the optimization procedure in Pyzgoubi for the racetrack lattice. The original and optimized DA points are shown by white points and a white arrow indicating the direction of optimization. 


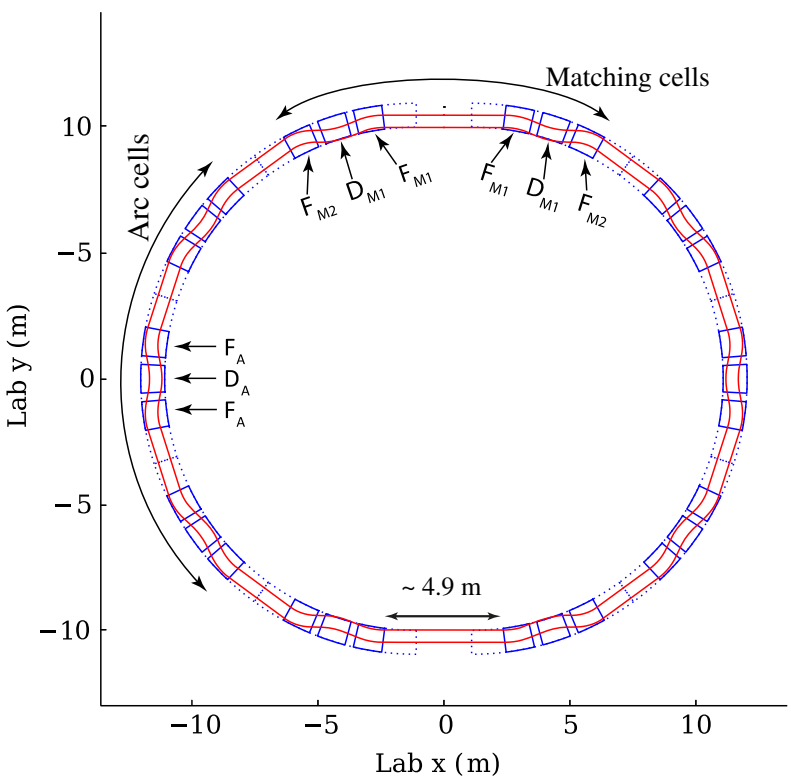

FIG. 15. A schematic of the NORMA racetrack lattice with $L_{R T}=2.0 \mathrm{~m}$, showing the lowest (inner) and highest (outer) energy orbits in red. Magnets are outlined by solid blue lines and the cell boundaries as dashed blue lines. Note that the solid blue outlines indicate only the approximate radial positions and horizontal aperture of the magnets, however the sector width of each is accurate. The "arc" and "matching" type cells are indicated as well as the five families of associated magnets.

variation from the ideal at $100 \mu \mathrm{m}$ is given in Table V. The COD amplification factors are lower in the racetrack than in the ring, possibly due to the increased size of the radial magnetic field ( $\sim 49 \mathrm{~cm}$ as opposed to $\sim 43 \mathrm{~cm})$, hence the sensitivity to misalignment errors is reduced. The DA

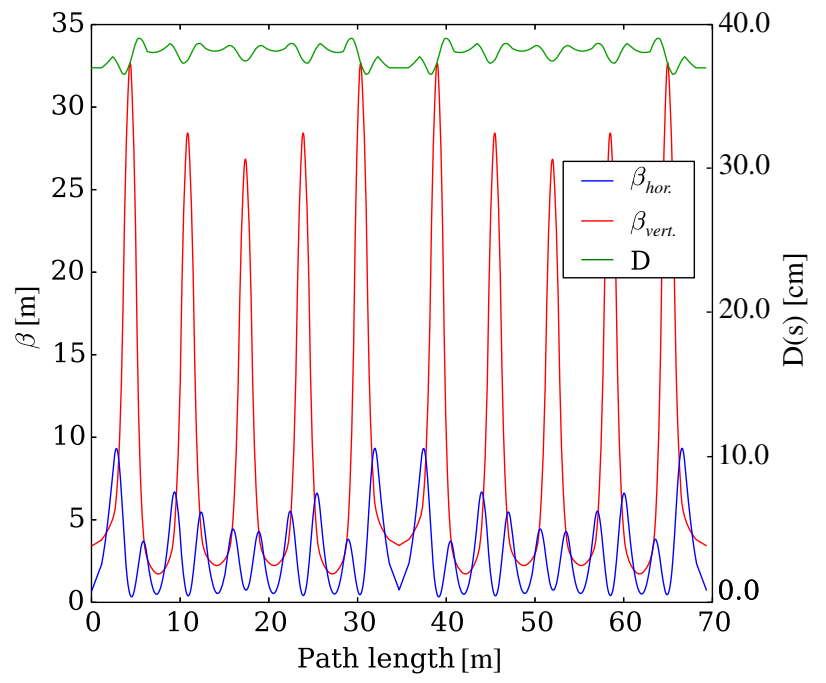

FIG. 16. The horizontal and vertical $\beta$-functions and dispersion at the injection energy of $30 \mathrm{MeV}$ are shown. The long straight racetrack section occurs just after $30 \mathrm{~m}$.

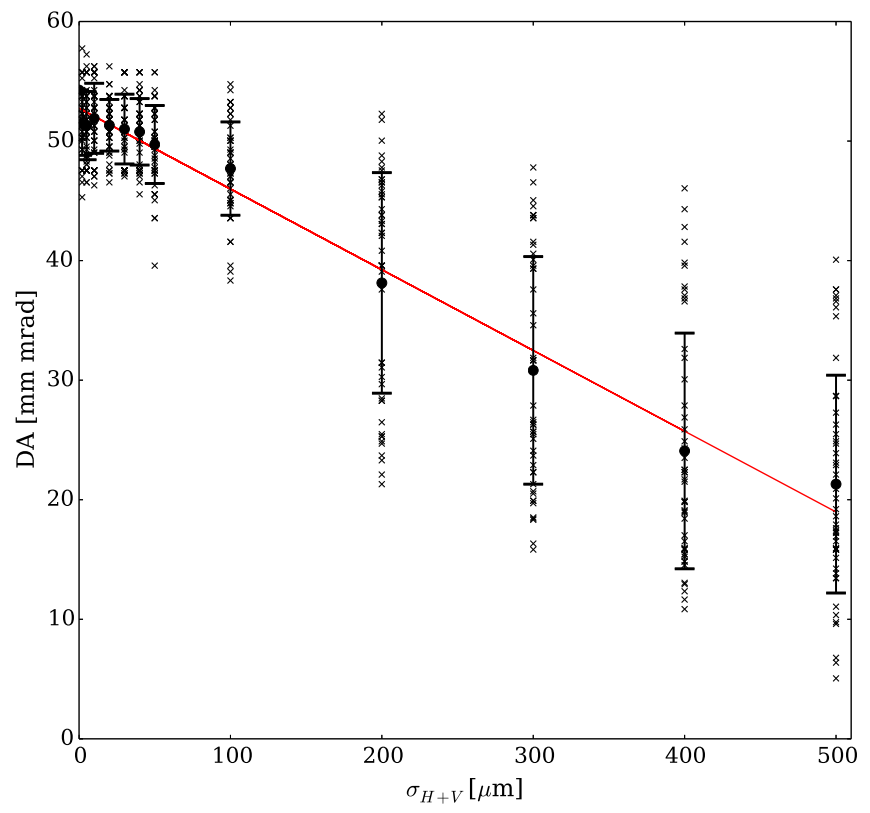

FIG. 17. The DA as a function of horizontal and vertical cell misalignment errors for the NORMA racetrack lattice with $f_{R T}=$ 0.91 and $L_{R T}=2.2 \mathrm{~m}$. The red line shows a minimum chisquared fit to the average reduction in DA with $\sigma$.

reduction factor is also slightly lower in the racetrack, possibly for the same reason. The variation in the tune over the energy range (of 0.02 and 0.0045 in the horizontal and vertical, respectively) is larger in the racetrack than in the ring, which is due to the violation of the perfect scaling law by inserting longer straight sections and breaking the symmetry.

\section{B. Concluding remarks-NORMA racetrack lattice}

We described a method for designing and optimizing a normal conducting racetrack lattice using the NORMA ring as a starting point. Due to the strong focusing into the long straight sections, the field in the $F_{M 1}$ magnets increases to $>1.8 \mathrm{~T}$ when $L_{R T}>1.0 \mathrm{~m}$. We therefore presented one solution to this problem in order to keep the field below $1.8 \mathrm{~T}$ and maintain a stable racetrack with a good DA, by scaling the size and fields in the lattice.

We presented an example of a scaled normal conducting racetrack lattice using our methodology which has a total magnet-free drift straight of $4.9 \mathrm{~m}$. The average horizontal orbit excursion was $0.49 \mathrm{~m}$ (maximum $0.5 \mathrm{~m}$ in the center of the F magnet), the circumference was $70.7 \mathrm{~m}$ and the DA was $57.7 \mathrm{~mm}$ mrad for an ideal lattice, and remained above $40 \mathrm{~mm}$ mrad with up to $100 \mu \mathrm{m}$ of transverse misalignment errors. We were therefore able to optimize a racetrack lattice that meets the same clinical requirements as the ring for use in pCT. Additionally, it has a larger area of magnetfree drift that can be used to facilitate easier injection and extraction systems. 


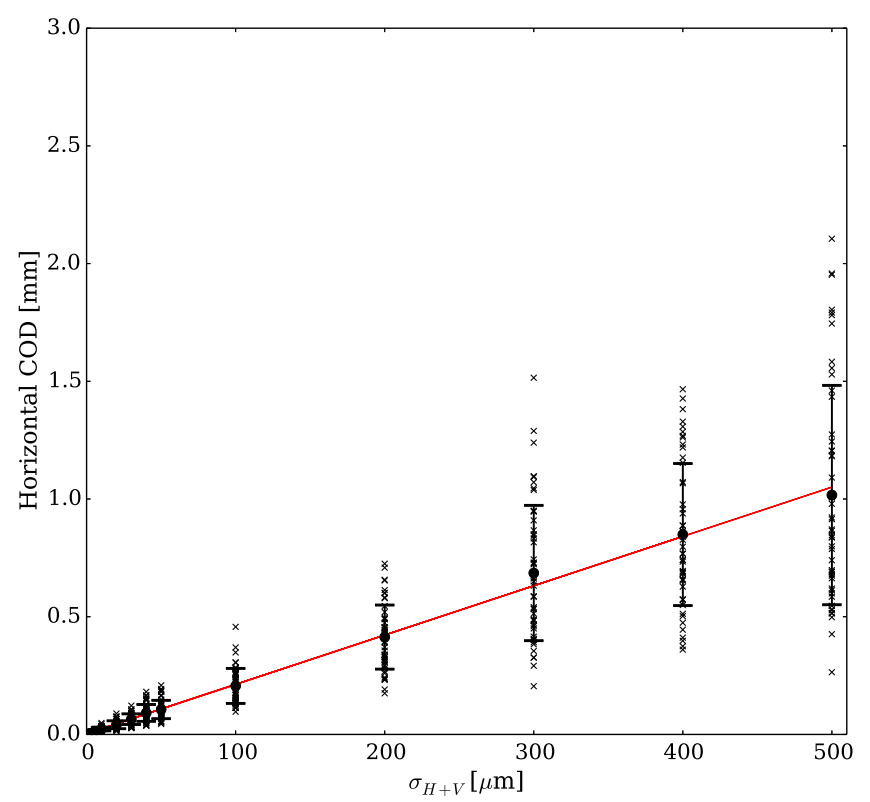

(a)

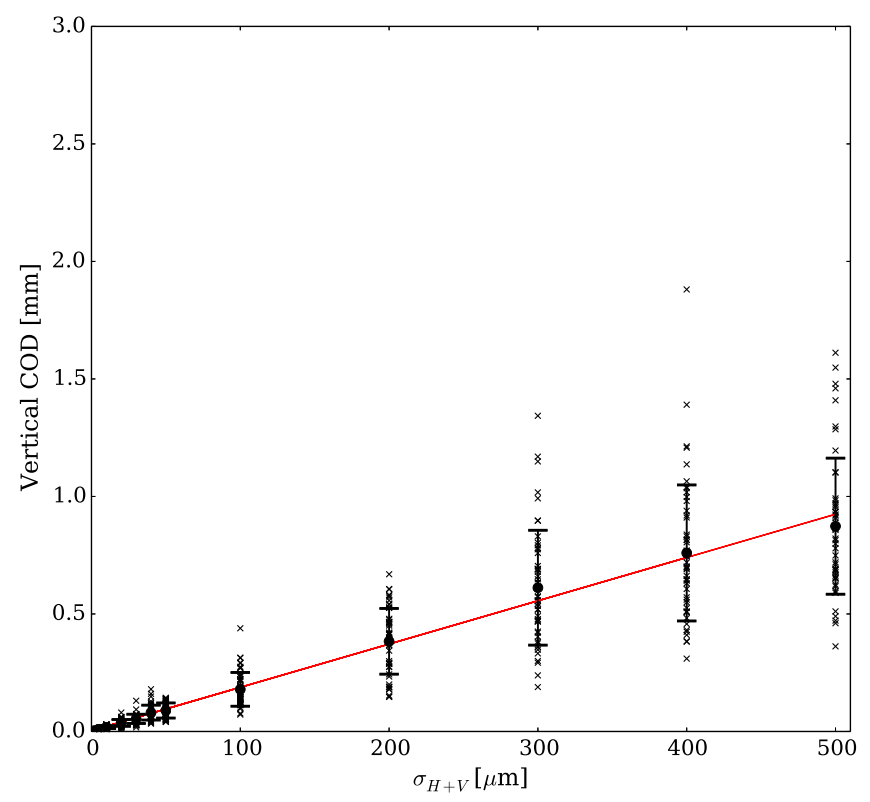

(b)

FIG. 18. The horizontal (a) and vertical (b) COD with cell misalignment errors for the racetrack lattice. Each individual randomly offset lattice is shown as a cross, the mean is shown as a filled circle, and the standard deviation as the error bars. The red line shows a minimum chi-squared fit to the data.

\section{RADIO FREQUENCY ACCELERATION, INJECTION, AND EXTRACTION}

In common with the previous PAMELA design study [31], we are motivated to supply pulse-by-pulse variation of the extracted bunch energy between $30 \mathrm{MeV}$ and $350 \mathrm{MeV}$ to allow multibunch painting and good uniformity of the delivered dose; hence an acceleration cycle of $1 \mathrm{~ms}$ is specified. Injection is envisaged to be from a conventional
TABLE V. A comparison of the COD amplification factors, DA reduction factor and rms tune variation from the mean for $100 \mu \mathrm{m}$ misalignment errors for the ring and racetrack lattices. $A_{H}$ and $A_{V}$ refer to the horizontal and vertical amplification factors, respectively.

\begin{tabular}{lcc}
\hline \hline Parameter & Ring & Racetrack \\
\hline$A_{H}$ & 3.0 & 2.1 \\
$A_{V}$ & 3.5 & 1.8 \\
DA reduction factor & 74.3 & 67.5 \\
Horizontal ring tune variation at $100 \mu \mathrm{m}$ & $3.9 \times 10^{-4}$ & $1.5 \times 10^{-2}$ \\
Vertical ring tune variation at $100 \mu \mathrm{m}$ & $3.7 \times 10^{-4}$ & $1.4 \times 10^{-3}$ \\
\hline \hline
\end{tabular}

$30 \mathrm{MeV}$ cyclotron-for example the IBA Cyclone-30 [53,54] or ACSI TR-30 [55] designs-such that the frequency of bunches from the cyclotron is not synchronous with the frequency of the FFAG; this is discussed below. Emittances from such cyclotrons can be less than $10 \mathrm{~mm} \mathrm{mrad}$, so that a lattice transverse acceptance greater than $40 \mathrm{~mm}$ mrad will be sufficient to efficiently capture the cyclotron bunches upon injection into the FFAG.

We consider the use of ferrite-loaded rf cavities as proposed for PAMELA, in which each $1.1 \mathrm{~m}$-long cavity provides two accelerating gaps each with a gap voltage up to $15 \mathrm{kV}$ peak. Eight $2.4 \mathrm{~m}$ short straight sections in either the circular or racetrack version of NORMA are thus sufficient to incorporate 16 cavities to provide up to $480 \mathrm{keV}$ of acceleration per turn. Allowing that the synchronous phase $\phi_{s} \sim 70$ degrees, this means that $400 \mathrm{keV}$ per turn may be comfortably obtained from such a system; we assume more conservatively an energy gain of $350 \mathrm{keV}$ per turn, which means an acceleration time of $0.5 \mathrm{~ms}$ or equivalently 910 turns. The turn separation of around $0.5 \mathrm{~mm}$ per turn is therefore much smaller than the typical beam size of several millimetres, and will remain smaller even for rather small injected emittances of $\sim 1 \mathrm{~mm}$ mrad. Hence, despite the horizontal orbit excursion of $\sim 450 \mathrm{~mm}$ being about 2.5 times larger than it would be in PAMELA, the shift is not large enough to use a cyclotron-like injection and extraction scheme, i.e., by using a dedicated extraction channel at a given radius.

TABLE VI. NORMA injection and extraction parameters.

\begin{tabular}{lc}
\hline \hline Parameter (unit) & Value \\
\hline Injection energy [MeV] & 30 \\
Extraction energy range [MeV] & $50-350$ \\
Kicker length [mm] & 500 \\
Kicker field [mT] & 30 \\
Injection kicker bend angle [mrad] & 38 \\
Extraction kicker bend angle [mrad] & $>10$ \\
Septum length [mm] & 1000 \\
Septum field [mT] & 500 \\
Injection septum bend angle [mrad] & 630 \\
Extraction septum bend angle [mrad] & $>160$ \\
\hline \hline
\end{tabular}


Similar to PAMELA, a pulsed extraction method is required, wherein the beam is extracted at a given time rather than at a given radius.

The orbit frequency of the circular machine varies from 1.266 to $3.328 \mathrm{MHz}$, a frequency shift of 2.6:1 which is similar to the 2.4:1 frequency shift of PAMELA. We aim to adopt a harmonic number of $h=14$ to give a cavity operating frequency from 17.7 to $46.6 \mathrm{MHz}$ in the circular machine, and a similar frequency range in the racetrack option. We therefore may adopt a similar ferrite-loaded cavity design to that developed for PAMELA, which itself is an adaptation of the existing cavities utilized in the UK ISIS synchrotron [56]; the cavity power required by the rf system will be around $1 \mathrm{MW}$, a little higher than in conventional therapy facilities but not a value that will be prohibitive. However we acknowledge that there may be some difficulty in realizing such a cavity for NORMA given the relatively large horizontal orbit excursion over the momentum range, which is roughly 2.5 times that of PAMELA for example.

\section{A. Injection from cyclotron}

Whilst NORMA may receive protons from a suitable $30 \mathrm{MeV}$ preinjector and linac, it is likely to be more costeffective to utilize a cyclotron. A similar injector method is used in the "cyclinac" scheme $[57,58]$ in which a cyclotron provides particles to a high-gradient linac, and it is well known that the cyclotron bunch extraction frequency (typically in the range $40-80 \mathrm{MHz}$ ) is not at all matched to the typical adopted S-band linac frequencies around $3 \mathrm{GHz}$. The cyclotron bunches are both asynchronous with the linac rf and rather long, typically several nanoseconds in comparison to the $0.33 \mathrm{~ns}$ linac bunch rate, so that the overall transmission of fully accelerated bunches at the end of the linac is inherently limited to less than around $10 \%$ both by the longitudinal capture and by transverse collimation. However, the $\sim 90 \%$ loss of particles in the linac is mostly compensated for by gating the bunches from the cyclotron as closely as possible to the $\sim 1 \mu$ s rf pulse length generated in the linac at $\sim 400 \mathrm{~Hz}$.

A similar method of injection to that of the cyclinac may be used in NORMA. In the NORMA case an injection kicker will provide an effective time window for injection of about 1 bunch out of the 14 buckets that encompass an orbit time of around $700 \mathrm{~ns}$; the incoming cyclotron bunches will be spaced $\sim 10 \mathrm{~ns}$ apart with typical lengths of a few nanoseconds (depending on the path length from cyclotron to FFAG; this introduces some ballistic spreading of the extracted cyclotron bunches due to their energy spread that is somewhat less than $1 \mathrm{MeV}$ ). The injected bunches will be somewhat shorter than the $18 \mathrm{MHz}$ rf buckets, so that charge from several cyclotron bunches may be captured into the chosen FFAG bucket; that captured bunch is then accelerated after the injection kicker pulse. Gating of the cyclotron is probably best achieved in a similar manner to that used in the Varian superconducting cyclotron $[59,60]$, where an electrostatic deflector plate near to the ion source steers unwanted, low-energy protons into the vacuum walls with a time response less than $50 \mu \mathrm{s}$; combined with pulsing of the ion source itself (which may be gated in windows typically around $1 \mathrm{~ms}$ ), losses and consequent activation of the FFAG can be limited.

For proton therapy we must deliver $\sim 10^{11}$ protons per fraction, or on average around $2 \times 10^{9}$ protons per second (pps) (assuming 1 Gy of dose is delivered to a liter volume in 1 minute) with a maximum proton flux of around $10^{10} \mathrm{pps}$. At a $1 \mathrm{kHz}$ pulse repetition rate this is around $10^{7}$ protons per pulse (ppp) or $1.6 \mathrm{pC}$ bunch charge. Space charge effects are therefore expected to be small; bunch charges in medical synchrotrons are much larger (by about a thousand times).

A $30 \mathrm{MeV}$ cyclotron may readily obtain $100 \mu \mathrm{A}$ average extracted current $[53,55]$, the extracted bunch rate being around $65 \mathrm{MHz}$ (if for example the cyclotron is operated at an $h=4$ harmonic with a guide field of about $1 \mathrm{~T}$ ). This corresponds to at least $\sim 10^{7} \mathrm{ppp}$, which is sufficient for rapid treatment. Gating the ion source emission to match it to the capture window of the FFAG will restrict the average current loss during FFAG injection to around $0.1 \mu \mathrm{A}$, or 3 Watts. This loss rate will cause some activation in the components, but it is about one hundred times smaller than the typical losses incurred when degrading the $\sim 1 \mu \mathrm{A}$, $230 \mathrm{MeV}$ extracted protons obtained in currently operating cyclotron-based treatment facilities. The number of protons required for imaging is much smaller than for a treatment fraction-perhaps a thousand times fewer-and to obtain a suitable tomograph the image acquisition time is limited in part by the requirement to rotate the gantry through which the high-energy protons are imparted to the patient. Typical gantry rotation speeds are around $1 \%$ s so that the imaging time will not be less than a minute; the proton fluence needed for imaging will therefore be about one thousand times less than that for treatment, and so not relevant from the point of view of activation. However, proton imaging will require a small, controllable proton fluence, and we envisage this may be achieved by reducing the ion source current of the cyclotron.

\section{B. Acceleration and extraction from FFAG}

The predicted DA of over $40 \mathrm{~mm}$ mrad is thought sufficient to efficiently accelerate each proton bunch without significant loss, and we expect any loss to occur upon extraction. Given that the average current in the FFAG is then the same as that delivered to the patient-about $0.1 \mathrm{nA}$-we do not expect any different activation or radiation protection issues to those encountered in other types of therapy facility. Extraction is more challenging than injection due to the much larger maximum beam rigidity, but the racetrack lattice long straight length of 
$4.9 \mathrm{~m}$ facilitates the use of single-straight schemes for both extraction and injection.

Our single-straight scheme envisages a kicker/septum combination in each long straight. We propose to utilize a conservative kicker field strength of $30 \mathrm{mT}$ so that a kicker pulse rise/fall time of $50 \mathrm{~ns}$ may be readily achieved and which - in the case of injection - means that transient fields after the kicker pulse are minimized. At the highest energy the kicker field translates the beam $15 \mathrm{~mm}$ across the septum entrance (the kicker center would be located $1.5 \mathrm{~m}$ from the septum entrance), which allows a $3 \mathrm{~mm}$ septum thickness to be used whilst also giving some margin for alignment errors.

A septum field of $500 \mathrm{mT}$ is also straightforwardly achieved, and allows the extracted bunches to clear the downstream ring bending magnet. We envisage the use of a fixed full-aperture kicker, and a moveable extraction septum magnet; the injection septum is fixed and the combined kicker and septum bend angles readily allow injection from a cyclotron sited within the FFAG circumference. The extraction septum magnet will move over $400 \mathrm{~mm}$ across the beam aperture to allow variable energy extraction [61-63], and thereby becomes the limitation on the rate of energy variation. A summary of our proposed injection and extraction parameters is given in Table VI. However, such septa may move fast enough to allow layer scanning at several $\mathrm{MeV}$ per second. A benefit of a moveable septum is that it allows extraction at rather low energies, the lower limit corresponding to the amount of aperture required for the first few turns of injected beam. The lower limit of extracted energy may therefore be as little as around $40 \mathrm{MeV}$, lower than is readily achieved in cyclotrons or synchrotrons. For example, the lower energy limit of isochronous cyclotrons is determined by what loss is tolerable when degrading from the fixed cyclotron extraction energy to the lowest energy; this limit is around $70 \mathrm{MeV}$.

Although the extracted beam position at the septum entrance may vary by nearly $400 \mathrm{~mm}$, lower-energy bunches are bent more by the septum field so that a natural focus will be formed downstream in the extraction line; a second kicker magnet might be necessary in the extraction line to allow correction of the beam trajectory through the downstream septum and beam transport. The downstream beam transport system optics will follow the change of energy in a manner similar to that proposed for variable-energy extraction in other FFAG and cyclotron proposals $[31,63]$.

\section{CONCLUSIONS}

We have presented our designs for a $30-350 \mathrm{MeV}$ normal conducting scaling FFAG for proton therapy. The ability to reach $350 \mathrm{MeV}$ allows such machines to realize pCT which is recognized by the clinical community to be extremely important when performing a precise voxel scan of a tumor in order to irradiate a hard-edged profile and ensure no healthy tissue is damaged. Using an FFAG enables rapid acceleration to take place over the energy range, allowing rapid voxel scanning, repainting and depth control which are also highly desired clinically. Our normal conducting design will allow for magnets which are potentially cheaper and simpler to operate when compared to superconducting ones, and although larger will inherently present good tolerance to misalignment errors. The ring and racetrack designs have circumferences of 60.4 and $70.7 \mathrm{~m}$, respectively, where the ring circumference is comparable to the PAMELA carbon ring of circumference $\sim 60 \mathrm{~m}$ and the racetrack is around 14\% larger. Although our racetrack design is larger than the PAMELA design, there are potential benefits in cost and operation arising from the normal conducting magnet designs.

An optimization procedure was written and developed using pyzgoubi to allow the optimization of a ring or racetrack FFAG given specific constraints such as geometry and working point. We wish to make clear to the reader that the flexibility of the optimization procedure allows it to be extended to any type of lattice design using pyzgoubi and that this procedure is not commonly described in the same detail in most lattice design work in the literature. Our computationally intensive techniques also allow us to carry out high precision calculations, which enabled us to optimize our working point with a high degree of accuracy.

The NORMA ring design has an average horizontal orbit excursion over the energy range of $43 \mathrm{~cm}$ (maximum $44 \mathrm{~cm}$ in the center of the F magnet), a DA of $68 \mathrm{~mm}$ mrad in an ideal lattice with a horizontal magnet aperture of $\sim 0.5 \mathrm{~m}$ and a length of $\sim 1.0 \mathrm{~m}$-roughly double that of the PAMELA design. We have demonstrated how this ring lattice may be extended into a racetrack configuration by optimizing in pyzgoubi and given an example of a racetrack with $\sim 4.9 \mathrm{~m}$ of magnet-free drift space. The design has an average horizontal orbit excursion of $49 \mathrm{~cm}$ (maximum $50 \mathrm{~cm}$ in the center of the F magnet) and a DA of $58 \mathrm{~mm} \mathrm{mrad}$ in the ideal lattice. The larger racetrack drift spaces allow for simpler injection and extraction modules to be designed.

It was also demonstrated that $L_{R T}$ may be increased beyond $2.0 \mathrm{~m}$ where such a lattice may be optimized in Pyzgoubi for a higher DA, however the field seen by a particle with $350 \mathrm{MeV}$ increases above $1.8 \mathrm{~T}$ and we must scale the lattice appropriately in this case, as described. Both the ring and racetrack lattices have a good tolerance for magnetic misalignments up to $100 \mu \mathrm{m}$ which allow the DA to remain $>40 \mathrm{~mm}$ mrad and the COD $<0.5 \mathrm{~mm}$.

Work is currently being carried out to design a 3D field map for the NORMA magnets such that realistic bunches may be tracked in Pyzgoubi, allowing us to refine and iterate over the design. A more comprehensive error study will also be carried out, including magnet rotations as well as transverse offsets. Additionally, a multipole model of the magnets is currently being created in Pyzgoubi in order to simulate individual multipole errors within the ideal scaling field that may occur in a realistic magnet which may also be compared to the field map design. The rf acceleration 
modules will also be considered in more detail as we acknowledge the challenges presented by a relatively large transverse aperture.

Figure 12 shows that it is possible to design a racetrack lattice in our parameter range with $L_{R T} \approx 20 \mathrm{~m}$, however the DA falls quickly; we are currently studying this relationship in more detail. Throughout the current NORMA design in this paper, the field index $k$ is a constant for all magnet families. However we are beginning to study a racetrack in which we define different $r_{0}$ and $k$ in the matching sections compared to the arc sections. These extra degrees of freedom may allow us to better match the optics into a long straight section by allowing more flexible control over the $\beta$-functions and dispersion. This so-called "egg-shaped" design has already been proposed [64,65] and has been shown to allow flexibility in matching racetracks with long straight sections. This type of design may ultimately allow us to decrease the size of the arc sections in NORMA and increase the straight sections to accommodate some of the rf modules as well as injection/ extraction systems and possibly allowing us to shrink the accelerator footprint further.

\section{ACKNOWLEDGMENTS}

The authors would like to thank Suzie Sheehy (Rutherford Appleton Laboratory) and Franćois Méot (Brookhaven National Laboratory), for technical information, useful discussions and suggestions. We would also like to thank J. B. Lagrange and Jaroslaw Pasternak (both of Imperial College London) for their discussions on FFAG dynamics and for our ongoing collaborations in this field, and Jim Clarke and Kiril Marinov for their collaboration in the ongoing magnet designs for NORMA. This work supported by Science and Technology Facilities Council (STFC) Grant No. ST/K002503/1.

[1] H. Owen, D. Holder, J. Alonso, and R. Mackay, Technologies for delivery of proton and ion beams for radiotherapy, Int. J. Mod. Phys. A 29, 1441002 (2014).

[2] R. Wilson, Radiological use of fast protons, Radiology 47, 487 (1946).

[3] Ion Beam Therapy, edited by U. Linz (Springer Verlag, Heidelberg, 2005).

[4] H. Owen, R. MacKay, K. Peach, and S. Smith, Hadron accelerators for radiotherapy, Contemp. Phys. 55, 55 (2014).

[5] W. T. Chu et al., Lawrence Berkeley Laboratory Report No. LBL-33749, 1993.

[6] D. Trbojevic et al., in Proceedings of the 2nd International Particle Accelerator Conference, San Sebastiáán, Spain (EPS-AG, Spain, 2011), p. 2541.

[7] N. A. Harbi and S. Y. Lee, Design of a compact synchrotron for medical applications, Rev. Sci. Instrum. 74, 2540 (2003).
[8] Data from Particle Therapy Co-Operative Group, www .ptcog.ch, Accessed February 2015.

[9] Proton and Carbon Ion Therapy, edited by C.-M. Ma and T. Lomax (CRC Press, Boca Raton, FL, 2013).

[10] U. Schneider, E. Pedroni, and A. Lomax, The calibration of CT Hounsfield units for radiotherapy treatment planning, Phys. Med. Biol. 41, 111 (1996).

[11] M. van Herk, Errors and margins in radiotherapy, Semin. Radiat. Oncol. 14, 52 (2004).

[12] K. M. Hanson, J. N. Bradbury, T. M. Cannon, R. L. Hutson, D. B. Laubacher, R. J. Macek, M. A. Paciotti, and C. A. Taylor, Computed tomography using proton energy loss, Phys. Med. Biol. 26, 965 (1981).

[13] R.W. Schulte et al., Conceptual design of a proton computed tomography system for applications in proton radiation therapy, IEEE Trans. Nucl. Sci. 51, 866 (2004).

[14] C. Talamonti et al., Proton radiography for clinical applications, Nucl. Instrum. Methods Phys. Res., Sect. A 612, 571 (2010).

[15] R. W. Schulte, V. Bashkirov, M. C. L. Klock, T. Li, A. J. Wroe, I. Evseev, D. C. Williams, and T. Satogata, Density resolution of proton computed tomography, Med. Phys. 32, 1035 (2005).

[16] G. Coutrakon et al., A new proton CT scanner, arXiv:1409.0049.

[17] G. Poludniowski, N. M. Allinson, T. Anaxagoras, M. Esposito, S. Green, S. Manolopoulos, J. Nieto-Camero, D. J. Parker, T. Price, and P. M. Evans, Proton-counting radiography for proton therapy: A proof of principle using CMOS APS technology, Phys. Med. Biol. 59, 2569 (2014).

[18] U. Amaldi, A. Bianchi, Y.-H. Chang, A. Go, W. Hajdas, N. Malakhov, J. Samarati, F. Sauli, and D. Watts, Construction, test and operation of a proton range radiography system, Nucl. Instrum. Methods Phys. Res., Sect. A 629, 337 (2011).

[19] M. Scaringella et al. (The PRIMA (PRoton IMAging) collaboration), Development of a proton computed tomography apparatus, Nucl. Instrum. Methods Phys. Res., Sect. A 730, 178 (2013).

[20] M. Bucciantonio and F. Sauli, Proton computed tomography, Mod. Phys. Lett. A 30, 1540024 (2015).

[21] N. K. Abrosimov, Y. A. Gavrikov, E. M. Ivanov, D. L. Karlin, A. V. Khanzadeev, N. N. Yalynych, G. A. Riabov, D. M. Seliverstov, and V. M. Vinogradov, $1000 \mathrm{MeV}$ proton beam therapy facility at Petersburg Nuclear Physics Institute Synchrocyclotron, J. Phys. Conf. Ser. 41, 424 (2006).

[22] E. Vanzi et al., The PRIMA collaboration: Preliminary results in FBP reconstruction of pCT data, Nucl. Instrum. Methods Phys. Res., Sect. A 730, 184 (2013).

[23] K. R. Symon, D. W. Kerst, L. W. Jones, L. J. Laslett, and K. M. Terwilliger, Fixed-field alternating-gradient particle accelerators, Phys. Rev. 103, 1837 (1956).

[24] D. Trbojevic, FFAGs as accelerators and beam delivery devices for ion cancer therapy, Rev. Accel. Sci. Techol. 02, 229 (2009).

[25] Y. Sato et al., in Proceedings of the 11th European Particle Accelerator Conference, Genoa, Italy, 2008 (EPS-AG, Geneva, 2008), p. 581. 
[26] M. Aiba et al., in Proceedings of the Particle Accelerator Conference, Chicago, IL, 2001 (IEEE, New York, 2001), p. 3254.

[27] M. Tanigaki et al., in Proceedings of the 21st Particle Accelerator Conference, Knoxville, TN, 2005 (IEEE, Piscataway, NJ, 2005), p. 350.

[28] S. Machida et al., Acceleration in the linear non-scaling fixed-field alternating-gradient accelerator EMMA, Nat. Phys. 8, 243 (2012).

[29] F. Méot et al., in Proceedings of the 11th European Particle Accelerator Conference, Genoa, Italy, 2008 (EPS-AG, Geneva, 2008), p. 1791.

[30] S. Sheehy, K. Peach, H. Witte, D. J. Kelliher, and S. Machida, Fixed field alternating gradient accelerator with small orbit shift and tune excursion, Phys. Rev. ST Accel. Beams 13, 040101 (2010).

[31] K. J. Peach et al., Conceptual design of a non-scaling fixed field alternating gradient accelerator for protons and carbon ions for charged particle therapy, Phys. Rev. ST Accel. Beams 16, 030101 (2013).

[32] H. Witte et al., The advantages and challenges of helical coils for small accelerators; A case study, IEEE Trans. Appl. Supercond. 22, C2 (2012).

[33] T. Misu, Y. Iwata, A. Sugiura, S. Hojo, N. Miyahara, M. Kanazawa, T. Murakami, and S. Yamada, Design study of compact medical fixed-field alternating-gradient accelerators, Phys. Rev. ST Accel. Beams, 7, 094701 (2004).

[34] S. Machida, Scaling Fixed-Field Alternating Gradient Accelerators with a Small Orbit Excursion, Phys. Rev. Lett. 103, 164801 (2009).

[35] J. S. Berg, in Proceedings of the 8th European Particle Accelerator Conference, Paris, 2002 (EPS-IGA and CERN, Geneva, 2002), p. 1124.

[36] J.-B. Lagrange and Y. Mori, in Proceedings of the 23rd Particle Accelerator Conference, Vancouver, Canada, 2009 (IEEE, Piscataway, NJ, 2009), p. 4305.

[37] J.-B. Lagrange, T. Planche, and Y. Mori, Zero-chromatic FFAG straight section, Int. J. Mod. Phys. A 26, 1785 (2011).

[38] J.-B. Lagrange et al., in 5th International Particle Accelerator Conference, Dresden, Germany (JACoW, Geneva, Switzerland, 2014), p. 1208.

[39] H. Wiedemann, Particle Accelerator Physics, 3rd ed. (Springer, New York, 2007), p. 72.

[40] K. Marinov and J. Clarke (private communication).

[41] S. Tygier, R. B. Appleby, J. M. Garland, K. Hock, H. Owen, D. J. Kelliher, and S. L. Sheehy, PyZgoubi and the simulation of dynamic aperture in FFAGs, Nucl. Instrum. Methods Phys. Res., Sect. A 775, 15 (2015).

[42] F. Méot, The ray-tracing code Zgoubi, Nucl. Instrum. Methods Phys. Res., Sect. A 427, 353 (1999).

[43] A. G. Ruggiero, FFAG conference, TRIUMF, Vancouver B.C., Canada, http://hadron.kek.jp/FFAG/FFAG04_HP/, Accessed February 2015 (TRIUMF, Vancouver, BC, 2004).

[44] S. Koscielniak and M. K. Craddock, in Proceedings of the 9th European Particle Accelerator Conference, Lucerne, 2004 (EPS-AG, Lucerne, 2004), [http://accelconf.web.cern .ch/AccelConf/e04/], p. 1138.
[45] M. Tanigaki et al., in Proceedings of the 10th European Particle Accelerator Conference, Edinburgh, Scotland, 2006 (EPS-AG, Edinburgh, Scotland, 2006), p. 2367.

[46] H. A. Enge, Effect of extended fringing fields on ion-focusing properties of deflecting magnets, Rev. Sci. Instrum. 35, 278 (1964).

[47] J. M. Garland et al., in 5th International Particle Accelerator Conference, Dresden, Germany (JACoW, Geneva, Switzerland, 2014), p. 2198.

[48] (The SciPy Collaboration), http://www.scipy.org/, Accessed February 2015.

[49] J. A. Nelder and R. Mead, The downhill simplex method, Comput. J. 7, 308 (1965).

[50] S. Tygier, R. B. Appleby, J. M. Garland, K. Hock, H. Owen, D. J. Kelliher, and S. L. Sheehy, PyZgoubi and the simulation of dynamic aperture in FFAGs, Nucl. Instrum. Methods Phys. Res., Sect. A 775, 15 (2015), p. 20.

[51] University of Manchester (UK) EPS High Throughput Computing-Condor, http://condor.eps.manchester.ac.uk/, Accessed February 2015.

[52] S. Machida, in Proceedings of the International Particle Accelerator Conference, Kyoto, Japan (ICR, Kyoto, 2010), p. 558.

[53] J. Bol et al., in European Particle Accelerator Conference, Rome, Italy (World Scientific, Singapore, 1988), p. 1488.

[54] E. Forton et al., in 19th International Conference on Cyclotrons and Their Applications, Lanzhou, China (JACoW, Geneva, Switzerland, 2010), p. 189.

[55] V. Sabaiduc et al., in 18th International Conference on Cyclotrons and Their Applications, Giardini Naxos, Italy (JACoW, Geneva, Switzerland, 2007), p. 225.

[56] D. Findlay, in Proceedings of the 22nd Particle Accelerator Conference, PAC-2007, Albuquerque, NM (IEEE, New York, 2007), p. 695.

[57] A. Garonna, U. Amaldi, R. Bonomi, D. Campo, A. Degiovanni, M. Garlasché, I. Mondino, V. Rizzoglio, and S. V. Andrés, Cyclinac medical accelerators using pulsed $\mathrm{C}^{6+} / \mathrm{H}_{2}^{+}$ion sources, JINST 5, C09004 (2010).

[58] U. Amaldi et al., Cyclinacs: Fast-cycling accelerators for hadrontherapy, arXiv:0902.3533.

[59] M. Schillo et al., in Proceedings of the 16th International Conference on Cyclotrons and Their Applications (AIP, Melville, NY, 2001), p. 37.

[60] T. Stephani et al., in 19th International Conference on Cyclotrons and Their Applications, Lanzhou, China (JACoW, Geneva, Switzerland, 2010), p. 93.

[61] C. Baumgarten, Cyclotrons with fast variable and/or multiple energy extraction, Phys. Rev. ST Accel. Beams 16, 100101 (2013).

[62] F. Meot, A multiple-room, continuous beam delivery, hadron-therapy installation, Phys. Procedia 66, 361 (2015).

[63] S. Antoine et al., Principle design of a proton therapy, rapid-cycling, variable energy spiral FFAG, Nucl. Instrum. Methods Phys. Res., Sect. A 602, 293 (2009).

[64] J.-B. Lagrange et al., in 7th Annual Meeting of Particle Accelerator Society of Japan, Himeji, Japan (2010), p. 235.

[65] J. Pasternak et al., in Proceedings of the 2nd International Particle Accelerator Conference, San Sebastián, Spain (EPS-AG, Spain, 2011), p. 826. 